\title{
Controlled fabrication of ion track nanowires and channels
}

\author{
Reimar Spohr ${ }^{\text {a, b, }{ }^{*} \text {, Cristian Zet }}{ }^{\mathrm{d}}$, Bernd Eberhard Fischer ${ }^{\mathrm{b}}$, Helge Kiesewetter ${ }^{\mathrm{b}}$, Pavel Apel ${ }^{\mathrm{e}}$, Igor Gunko ${ }^{\mathrm{f}}$,
} Takeshi Ohgai $^{\mathrm{g}}$, Lars Westerberg ${ }^{\mathrm{b}}$

${ }^{a}$ Department of Physics and Materials Science, Uppsala University, Box 530, 75121 Uppsala (Sweden)

${ }^{\mathrm{b}}$ GSI Helmholtzzentrum für Schwerionenforschung GmbH, 64291 Darmstadt (Germany)

${ }^{c}$ Applied Physics, Universität Frankfurt, Max-von-Laue-Str. 1, 60438 Frankfurt (Germany

d Technical University "Ghe Asachi", Bd. D. Mangeron 53, 700050 Iasi (Romania)

e Joint Institute for Nuclear Research (JINR), 141980 Dubna (Russia),

${ }^{\mathrm{f}} \mathrm{RD \& G}$, Grundstr. 27, 64289 Darmstadt (Germany)

${ }^{\mathrm{g}}$ Nagasaki University, Bunkyo-Machi 1-14, Nagasaki 852-8521 (Japan)

* e-mail address of responding author: r.spohr@gsi.de

\begin{abstract}
We describe a system for fabricating prescribed numbers of ion track nanochannels and nanowires from a few hundred down to one. It consists of two parts: First, a mobile TAPE TRANSPORT SYSTEM, which, in connection with an ion beam from a heavy-ion accelerator (nuclear charge $\mathrm{Z}$ above 18 and specific energy between 1 and $10 \mathrm{MeV} /$ nucleon) tuned down to low flux density by means of defocusing and a set of sensitive fluorescence screens, can fabricate a series of equidistant irradiation spots on a tape, whereby each spot corresponds to a preset number of ion tracks. The tape transport system uses films of $36 \mathrm{~mm}$ width and thicknesses between 5 and $100 \mu \mathrm{m}$. The aiming precision the system depends on the diameter of the installed beam defining aperture, which is between 50 and $500 \mu \mathrm{m}$. The distance between neighboring irradiation spots on the tape is variable and typically set to $25 \mathrm{~mm}$. After reaching the preset number of ion counts the irradiation is terminated, the tape is marked and moved to the next position. The irradiated frames are punched out to circular membranes with the irradiation spot in the center. The second part of the setup is a compact CONDUCTOMETRIC SYSTEM with 10 picoampere resolution consisting of a computer controlled conductometric cell, sealing the membrane hermetically between two chemically inert half-chambers containing electrodes and filling/flushing openings, and is encased by an electrical shield and a thermal insulation. The ion tracks can be etched to a preset diameter and the system can be programmed to electroreplicate nanochannels in a prescribed sequence of magnetic/nonmagnetic metals, alloys or semiconductors. The goal of our article is to make the scientific community aware of the special features of single ion fabrication and to demonstrate convincingly the significance of controlled etching and electroreplication.

Keywords: conductometry, conductometric cell, electrodeposition, micro and nanofabrication, GMR, ion track technology, magnetoelectronics, nanowires, phase detection, real-time control, selforganization, sensor-technology, single-ion, spintronics, surfactant.

PACS 2010 Codes

81.07.-b Nanoscale materials and structures: fabrication and characterization

81.07.Gf Nanowires

81.15.Pq Electrodeposition, electroplating

81.16.-c Methods of micro- and nanofabrication and processing

81.20.-n Methods of materials synthesis and materials processing

85.75.-d Magnetoelectronics; spintronics: devices exploiting spin polarized transport or integrated magnetic field
\end{abstract}




\section{Contents}

1. Introduction 2

1.1. Ion track technology 2

1.2. Single ion techniques 3

1.3. From random to deterministic 3

1.4. Alternative techniques 4

1.5. Goal of this report 4

2. Piercing polymer foils with a preset number of ions 5

2.1. Ion beam requirements 5

2.2. Tape transport system 5

2.3. Low flux beam diagnostics 6

2.4. Performing an irradiation 7

2.5. Digital control system 8

2.6. User interface 8

3. Single ion track etching and replication 8

3.1. Conductometric system 8

3.2. Track replication 9

3.3. Computer interface 9

3.4. User interface 10

4. Single-ion track applications 10

4.1. Etching of single track 10

4.2. Etching of 100 tracks 11

4.3. Probability of double tracks 12

4.4. Modifying cone angle 12

4.5. Asymmetric single pores 13

4.6. Surfactant assisted etching 13

4.7. Magnetoresistance of homogeneous wires 14

4.8. Magnetoresistance of layered wires 15

4.9. Relevance of resistance for magnetic sensors 15

5. Conclusions and Outlook 16

6. Acknowledgement 16

References 17

\section{Introduction}

\subsection{Ion track technology}

Ion tracks are the result of the passage of swift heavy ions through solid matter and can be etched selectively in many- preferentially homogeneous and insulating - materials resulting in cones or channels. The smallest dimension corresponds to the size of the activated zone, which is between 6 and $20 \mathrm{~nm}$ and depends on the radiation sensitivity of the material. The smoothness of the etched track wall depends on the homogeneity of the used material which is related with its optical transparency. The range of materials which can be etched selectively after ion irradiation comprises minerals, glasses and many 
transparent polymers, among which polycarbonate (PC) and allyl diglycol carbonate (CR-39 $)$ are most widely used. A survey of materials is provided in the classical book by Fleischer, Price, and Walker [1]. Track studies with lengths between 1 and $100 \mu \mathrm{m}$ are common, usually based on ions heavier than argon in a specific energy range between 1 and $10 \mathrm{MeV} /$ nucleon. For relativistic ions even track lengths up to $700 \mu \mathrm{m}$ have been observed [2].

\subsection{Single ion techniques}

Ion tracks were discovered by D.A. Young in 1957 [3]. Their technological potential was soon recognized by Fleischer, Price and Walker [4] who applied the technique for fabricating homoporous biological filter materials [5] which stimulated branching-out research activities in many fields [1]. DeBlois and Bean [6] and Possin [7] performed the first experiments with single ion tracks. They selected single etched channels in thin pieces of muscovite mica containing few natural fission fragment tracks per sample. Artificial ion tracks became possible at cyclotrons [8-11] and linear accelerators [12]. The technique of single track irradiation was first developed at GSI where the combination of rapid beam switching with single ion detection opened the possibility to fabricate single track membranes predictably [13-16], starting with an aperture for a red cell deformability sensor (Fig 1, center) [17]. As the exact location of the ion track is uncritical for this sensor, a small circular aperture of $50 \mu \mathrm{m}$ diameter was used to place the ion on the polymer foil. For still smaller apertures slit scattering becomes rapidly dominant, increasing with $1 / r$, where $r$ is the aperture radius. Independently, at GSI an ion microbeam was developed [18], capable of placing individual ions with a precision of $1.3 \mu \mathrm{m}$ and demonstrated with a mass production of a regular pattern [19].

Individual nanochannels and nanowires resulting from single track irradiations have been used in a variety of fields, e.g. as weak links between adjacent superfluid volumes [20-21], as pH-sensitive, current rectifying, asymmetric apertures [22-23], and as Giant Magnetic Resistance based magnetic field sensors [24-27].

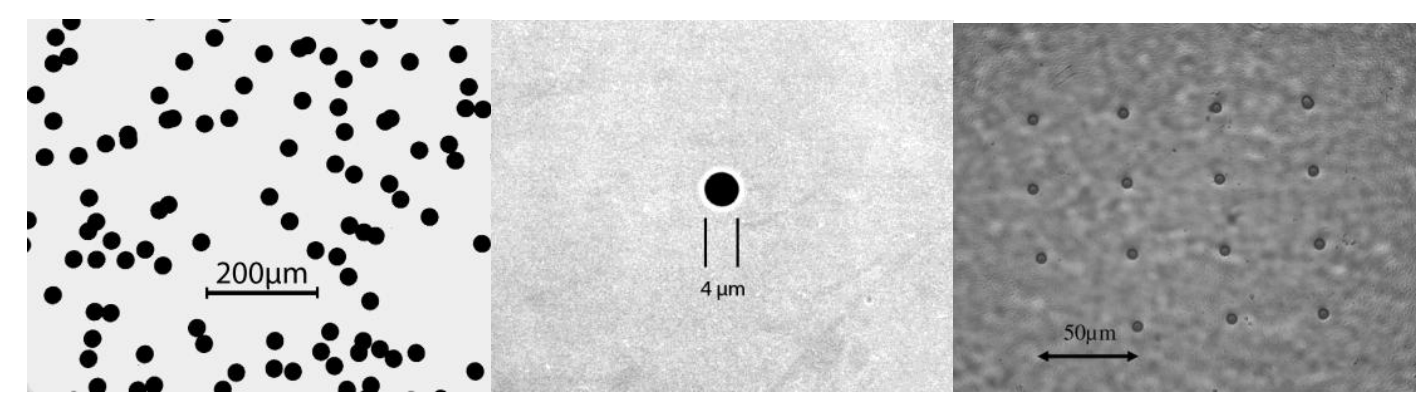

Fig 1 Random, single-ion, and microbeam ${ }^{2}$ irradiation.

\subsection{From random to deterministic}

The irradiation technique depends on the required spatial distribution of the ion tracks. Usually ion tracks are distributed at random. Random tracks are used to study and modify global properties. Randomness can be an advantage or a disadvantage, depending on the application. In filter applications the problem of multipore configurations exists and is tackled by wide angle irradiations. Fig 1 (left) shows a random distribution of about 100 etched cones of $32 \mu \mathrm{m}$ diameter in glass. At the given nominal porosity $P \approx 0.12$ (defined as the product of fluence $F$ and area $A$ of an individual etched track, where $F$ is the number of ions impinging per unit area), about 14 double and 1 triple track configurations were formed. Fig 1 center shows an etched channel of $4 \mu \mathrm{m}$ diameter, fabricated for red blood cell deformability studies. [17], [28] Fig 1 (right) shows a regular pattern of 15 single pores of about $5 \mu \mathrm{m}$

\footnotetext{
Trade name of PGG Industries, http://corporateportal.ppg.com/ppg/

2 Reprinted from Nuclear Instruments and Methods in Physics Research Section B, Vol 260, Fischer, B.E., Heiss, M., A facility to massproduce ordered single-hit patterns in continuous $35 \mathrm{~mm}$ films, 442-444, Copyright (2007), with permission from Elsevier
} 
diameter placed at distances of $50 \mu \mathrm{m}$. [19] Such irradiations require an ion microbeam and come close to the ideal of a fully deterministic irradiation. This type of aimed irradiation will not be dealt-with in this report.

\subsection{Alternative techniques}

Nanomaterials are being widely used in advanced technologies due to their unique properties inaccessible by other means. Among the variety of nanomaterials, materials containing nanochannels and nanowires attract increasing interest. First of all, this is caused by the revolutionary potential of the resistive-pulse sensing method that can be applied to detect and analyze important biological macromolecules [29-30]. As the sensitivity of the resistive-pulse technique increases with decreasing pore diameter and length, numerous approaches have been suggested to produce nanopores in thin foils, which are presented in recent review papers [31-32]. Another promising application of abiotic nanopores is to simulate the functions of biological channels formed by transmembrane proteins [33]. Recent studies performed on synthetic nanopores have provided valuable information on the transport of ions and neutral particles through the pores as small as some nanometers in diameter [34-35]. The knowledge of physical mechanisms governing the transport processes on the nanometer scale is expected to lead to integrated sample preparation and analysis systems [36]. Apart from individual nanopores, nanomaterials prepared by the so-called membrane based template synthesis [37] are also a subject of research and development activities in many laboratories. Focused electron and ion beams (FIB) are being used to fabricate pores down to diameters of a few nanometers [38]. Especially elegant are those methods which allow operating with a single nanopore or with a preset number of nanopores [38-39]. While FIB is particularly useful for thin membranes and pores of a few nanometers diameter, the ion track technique provides substantially higher aspect ratios up to about $10^{4}$. For building devices with precisely tunable mass transport, the ion track technique is a good choice because it offers the possibility to control in a wide range both the number of pores, the pore geometry and the aspect ratio [40].

We would like to highlight here two interesting alternatives to the single-ion fabrication technique, among which the single contact technique developed at EPFL [41] and the embedded membrane technique developed at GSI in collaboration with TU Munich [42-43] are most closely related with the work described here. First, the single contact technique uses multiporous ion track membranes. Both sides of the membrane are coated with a thin metal film. One metal film serves as cathode, the other metal film is placed closer to the anode and has a potential floating between anode and cathode potential. The wires grow at slightly different velocities from the cathode film toward the floating electrode film. As soon as the fastest growing wire contacts the floating film, its potential drop is used to terminate the electrodeposition process. In this way exclusively the resistance of the contacted single nanowire can be measured among numerous semi-completed nanowires. The technique can be applied to multiporous templates of alumina, polyimide, and polycarbonate. [44] Second, the embedded membrane technique is a combination of single-ion pores with self-assembled biopores [42]. It uses a large single-ion pore as a carrier frame to stabilize a lipid bilayer membrane acting as septum in a conductometric cell. One compartment of the conductometric cell contains subunits of self-assembling biological pores. As soon as the first pore has been self-assembled, the pore can be used to detect single and double stranded DNA fragments.

\subsection{Goal of this report}

The goal of our article is to make the scientific community aware of the special features of single ion channel fabrication and to demonstrate convincingly the possibility of controlled etching and electroreplication. We provide here an overview of available equipment and techniques to irradiate, etch and replicate individual ion tracks and describe the necessary equipment for fabrication of single-ion track micro- and nanostructures, including irradiation, etching and electroreplication. Etching can be interrupted as soon as the desired size is reached. The etched channel can be filled with another material 
by electrodeposition. The composition of the deposited material can be programmed and the electrodeposition can be interrupted at a prescribed wire length or bud diameter. Examples of fabricated channels and wires resulting from the described apparatus are provided. Our goal is to present the unique irradiation facilities offered by GSI and Uppsala and to provide a survey of applications in various fields, ranging from biosensors to spintronic devices.

\section{Piercing polymer foils with a preset number of ions}

\subsection{Ion beam requirements}

The energy of the incoming ion must be sufficient to penetrate the vacuum window, the tape, and the sensitive zone of the detector, whereby the flux density of the ion beam has to be reduced to be able to switch the ion beam reliably off after the arrival of one or a preset number of ions. Therefore we describe here the special requirements for preparing an ion beam with sufficiently reduced flux density.

For wide beam irradiations, such as in filter production, the beam is usually swept over the sample area at full intensity. This is not the case in single ion irradiations where the beam flux density has to be decreased by several orders of magnitude to ensure sufficient low count rate to switch the beam off after a precise number of ions have passed through the beam defining aperture and through the target material. For this purpose, the beam intensity of the accelerator needs to be reduced in steps. First, using a Faraday cup, the ion beam is decreased to an intensity below 1 particle nA corresponding to the lower limit of convenient current measurements. Second, using a series of fluorescent screens placed along the beamline as monitors, the intensity is decreased by about five orders of magnitude by defocusing the ion beam while keeping it on the prescribed beam-line axis. We use as fluorescent material $\mathrm{Al}_{2} \mathrm{O}_{3}, \mathrm{CdWO}_{4}$ and $\mathrm{ZnS}$ with respectively increasing sensitivities. When reaching the lower limit of the most sensitive screen $(\mathrm{ZnS})$, the detector pulse rate is checked and the defocusing is readjusted to a count rate compatible with the shutter speed. For single-ion irradiations we use a count rate of typically 10 ions per second. For a critical aperture of $0.4 \mathrm{~mm}$ diameter this corresponds to a flux density of roughly $10^{4}$ ions cm $\mathrm{cm}^{-2}$.

\subsection{Tape transport system}

The compact and mobile tape transport system (shown in Fig 2 and Fig 3) is usually attached to an ion accelerator (nuclear charge $\mathrm{Z}$ above $\sim 18$ and specific energy between 1 and $10 \mathrm{MeV} /$ nucleon) but can in principle be used even with a tiny fission fragment source. In contrast to its precursor, the tape transport system described here is independent of tape perforations ${ }^{3}$. The ion penetrates a thin film vacuum window ( $4 \mu \mathrm{m}$ polyethylene terephthalate, PET) separating the accelerator vacuum from the tape irradiation system which is at ambient pressure. After having detected a preset number of ions, the ion beam is switched off by an electrostatic deflector, which is part of the accelerator, the tape is marked by a needle printer and the polymer foil is shifted to a new position. The shutter time has to be short compared with the average time between ion counting events. A more detailed description is given in the following.

3 The first single-ion irradiation system at GSI used perforated $36 \mathrm{~mm}$ films for transport and retrieval of irradiation spots. 


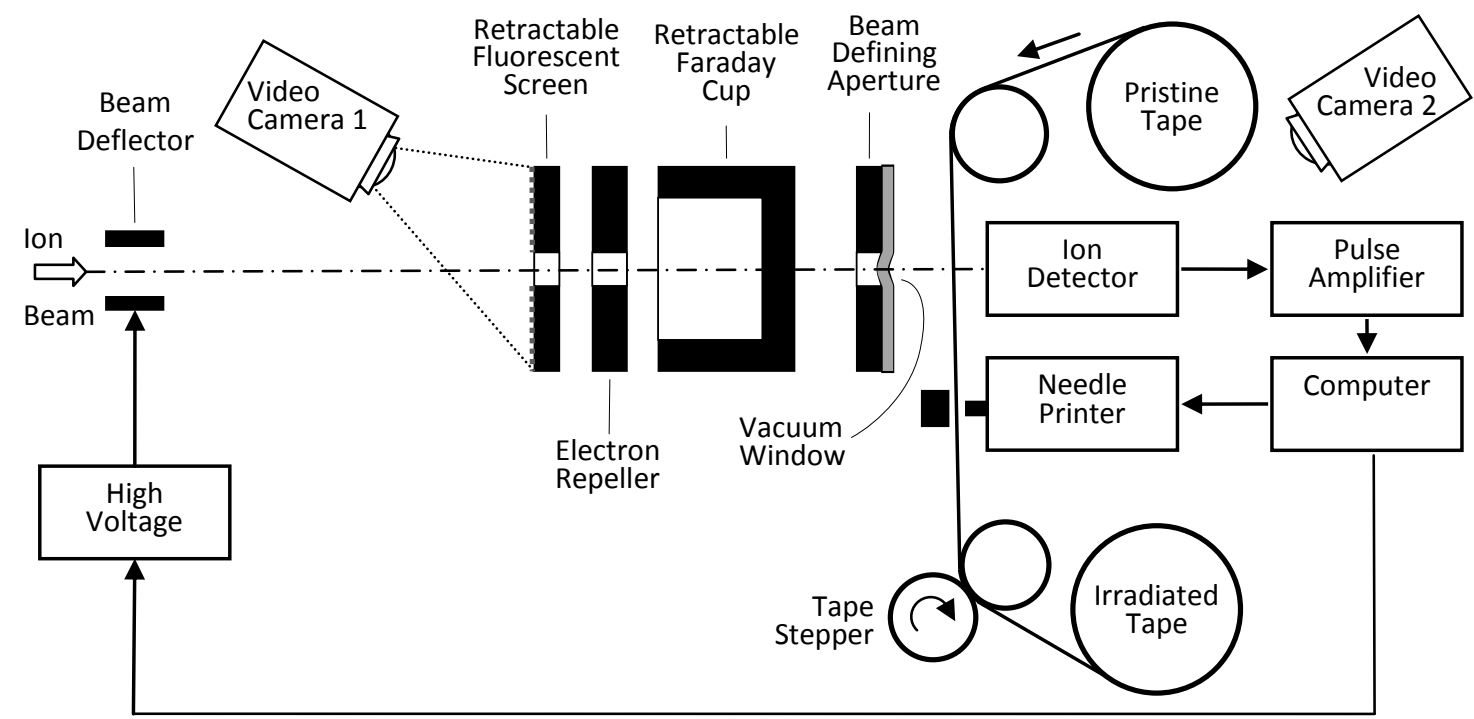

Fig 2 Principle of tape transport system ${ }^{4}$ consisting of beam deflector, beam diagnostics and tape transport. The high voltage supply and the beam deflector (left) are integral parts of the ion accelerator.

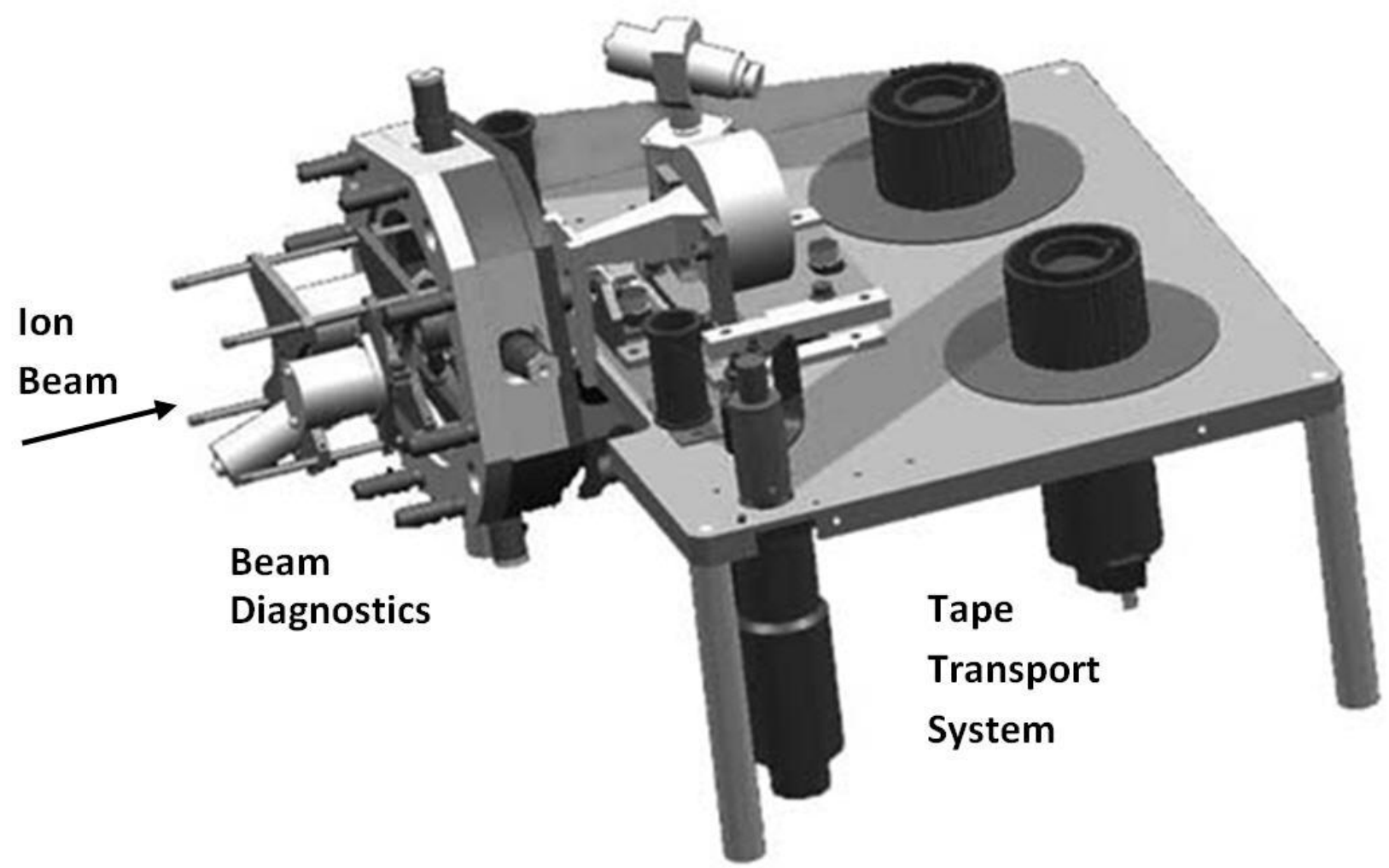

Fig 3 Perspective view of tape transport system. ${ }^{4}$ Left: beam diagnostics. Right: tape transport platform including a video camera for checking the tape transport, a marking indenter and ion detector (below the indenter). The tape transport platform can be attached at two fixed angles with respect to the beam axis corresponding to an ion incidence on the tape of $90^{\circ}$ (shown here) and $35^{\circ}$.

\subsection{Low flux beam diagnostics}

Video camera 1 is mounted inside the vacuum system and serves for observing the fluorescent screen continuously. The retractable fluorescent screen is the last but most sensitive screen in a series of three fluorescent screens arranged along the beam-line. It consists of a $\mathrm{ZnS}$ based phosphor which was

4 Developed at GSI Darmstadt for use at The Svedberg Laboratory of Uppsala University, Sweden; http://rsp.eunitt.de/ : Projects : Controlled Fabrication of Micro Channels : thumbnails 
paint-brushed on its backing plate from a suspension of the phosphor in an aqueous solution of sodium silicate, which serves as a binder. With this screen it is possible to observe ion beams down to about $10^{3}$ particles $\mathrm{cm}^{-2} \mathrm{~s}^{-1}$. The center bore of the fluorescent screen permits passing the beam through the electron repeller onto the retractable Faraday cup. The fluorescent screen has to be protected from rapid fading at beam intensities above 10 particle pA by retracting it from the ion beam. The electron repeller is used as the entrance aperture of a Faraday cup. It can be set on negative (repelling) potential with respect to the Faraday cup to prevent secondary electrons from escaping and falsifying the current measurement. The Faraday cup can be connected to a current digitizer or a pico-amperemeter and used for calibrating high beam intensities $>100$ particle $\mathrm{pA}$. It has to be inserted at high beam intensities to protect the ion detector from fading. The Faraday cup is withdrawn from the beam when reaching sufficiently low intensity for being able to use the fluorescent screen. The beam-defining aperture restricts the ion beam to a small spot on the polymer tape and is covered by a vacuum window consisting of a thin polymer foil $(4 \mu \mathrm{m}$ PET) attached to it by double adhesive from the outside of the vacuum system. To reduce ion scattering from the rim of the aperture we use apertures with rather large diameters between 50 and $500 \mu \mathrm{m}$.

\subsection{Performing an irradiation}

The tape transport uses guide rolls with tilted edges to keep the tape centered. The tape is kept under a defined mechanical tension by counteracting drive motors and its movement is remotely observed with Video Camera 2. After reaching the preset number of hits, the tape stepper roll pulls the tape to the next irradiation position. The irradiation points can be placed at preset distances along the axis of the polymer film. We use frame sizes of typically $25 \mathrm{~mm}$. As ion detector we use a silicon PIN diode from Hamamatsu ${ }^{5}$ with an active area of about $4 \times 4 \mathrm{~mm}^{2}$. In comparison to regular photo diodes, infrared sensitive PIN diodes have a large depletion depth and can be easily used for ion detection up to ion ranges between 20 and $30 \mu \mathrm{m}$ corresponding roughly to specific ion energies between 2 and $3 \mathrm{MeV} /$ nucleon. For thin tapes with thicknesses $d<50 \mu \mathrm{m}$ a polymer foil of approximate thickness $d^{\prime} \sim(50-d) \mu \mathrm{m}$ was placed in front of the tape to reduce the energy of the incident ion. In this way, the PIN diode could be used as an energy sensitive detector even for thin tapes. The needle printer marks the irradiation spot at the margin of the tape by an indention visible to the naked eye. After completing the tape irradiation, the indention is used for punching out circular disks of 18 or $25 \mathrm{~mm}$ diameter from the tape, whereby the punching tool is placed with respect to the indention. In this way circular disks are obtained containing the required number of ion tracks in their center. Irradiation frames containing the preset number of ion tracks within a preset pulse height window are marked as good by a broad indent ( 9 pins). All other irradiation frames, usually less than $1 \%$ of the irradiations, are marked as bad by a 1 pin indent and can be recognized and discarded during the punching.

Bad irradiations can be the result of shutter delay or random coincidences. They can also result from particles scattered at the rim of the beam defining aperture, arriving at the detector with reduced energy. In principle, the probability of bad events can be further suppressed by a coincidence arrangement consisting of two detectors, a thin film $\Delta E$ detector placed before the tape combined with an $E$ detector placed after the tape. Toward this goal, a thin film $\Delta E$ detector was developed by Thungström et al. [45] and tested but not used hitherto.

The detector signal is fed to an amplifier stage and shaping filter. The electronic system was tested with a separate PIN diode irradiated by a weak alpha source [6].The pulse height distribution of a tape irradiation is shown in Fig 4.

\footnotetext{
5 Si PIN photodiode S1223 series, Hamamatsu Photonics K.K. - Solid-state division, Japan, Cat. No KSPD0001E01, Jan. 2002; http://www.hamamatsu.com/

6 Cf-252, http://www.ornl.gov/sci/isotopes/__cf252.html
} 
Fig 4 Pulse height distribution of single-track detector using ${ }^{129} \mathrm{Xe}^{27+}$ ions of $8.3 \mathrm{MeV} /$ nucleon passing through a window of ca. $4 \mu \mathrm{m}$ PET and a $30 \mu \mathrm{m}$ thick PET tape tilted at $35^{\circ}$ (total track length ca. $48 \mu \mathrm{m}$ ).

\subsection{Digital control system}

The tape irradiation is controlled by a Field Programmable Gate Array (FPGA) [16] controlled via Ethernet from a host computer. The FPGA opens the beam shutter, digitizes the detector pulses, determines pulse height and counts the pulses. The ion detector is placed as close as possible behind the polymer film. The detector pulses are amplified by a preamplifier followed by a signal shaper and a fast analog to digital converter. The signal is amplified and shaped in order to minimize the influence of noise and pulse tail. After reaching the preset number of pulses, the digital pulse processor closes the beam shutter and shifts the tape to a new position. The detector efficiency is monitored during the tape irradiation. Detector degradation is characterized by gradually decreasing pulse height. The effect becomes important for very high total counts deposited within the irradiated spot on the ion detector. No apparent detector degradation was found up to $10^{4}$ pulse counts within a spot size of about $0.5 \mathrm{~mm}$ diameter, corresponding to a fluence of $5 \cdot 10^{6} \mathrm{ions} / \mathrm{cm}^{2}$.

\subsection{User interface}

The tape transport is controlled by a LabVIEW ${ }^{7} \operatorname{code}^{8}$. A module settings is used for setting the parameters of a data acquisition card, which provides eight digital input/output lines and two counters. A module estimates is used to estimate the number of available frames on the tape from the roll diameter and the tape thickness. The total irradiation time is estimated from the number of frames irradiated per second and the total number of frames to be irradiated. A module count rate is used for adjusting the average count rate to a value compatible with the times needed for pulse processing, shutter speed and transport time. We use typically 10 counts per second for single-ion irradiations. A module pulse shape permits to view the pulse shape of individual pulses, to adjust pulse amplification and - if necessary - to add tape thickness for reducing the pulse height to a value compatible with the detector thickness. The occurrence of coincident events can be minimized by reducing the ion beam intensity. A module irradiate is used to set the number of ions per frame and the total number of frames to be irradiated. For each irradiation a record is saved on hard disk.

\section{Single ion track etching and replication}

\subsection{Conductometric system}

We use a thermostated, thermally insulated, electrically shielded conductometric cell [46] inspired by contacts between Dubna and GSI [9] (Fig 5) in connection with a sensitive current amplifier for controlled etching. The two cell halves are machined of polychlorotrifluoroethylene (PCTFE) with

7 http://www.ni.com

8 http://rsp.eunitt.de : Projects : Controlled fabrication of Micro Channels : Thumbnails : References, Downloads, Links : e-cell Program 
polished flat ends for improved sealing of the membrane and are compressed by a spring loaded tightening screw. The electrodes and filling/flushing tubes are accessible during operation through a slit. The electrolyte can be removed automatically after reaching a desired pore diameter / wire length / bud diameter. The electric current is recorded as function of time and - after calibration - yields a direct reading of the actual channel diameter / wire length / bud diameter.

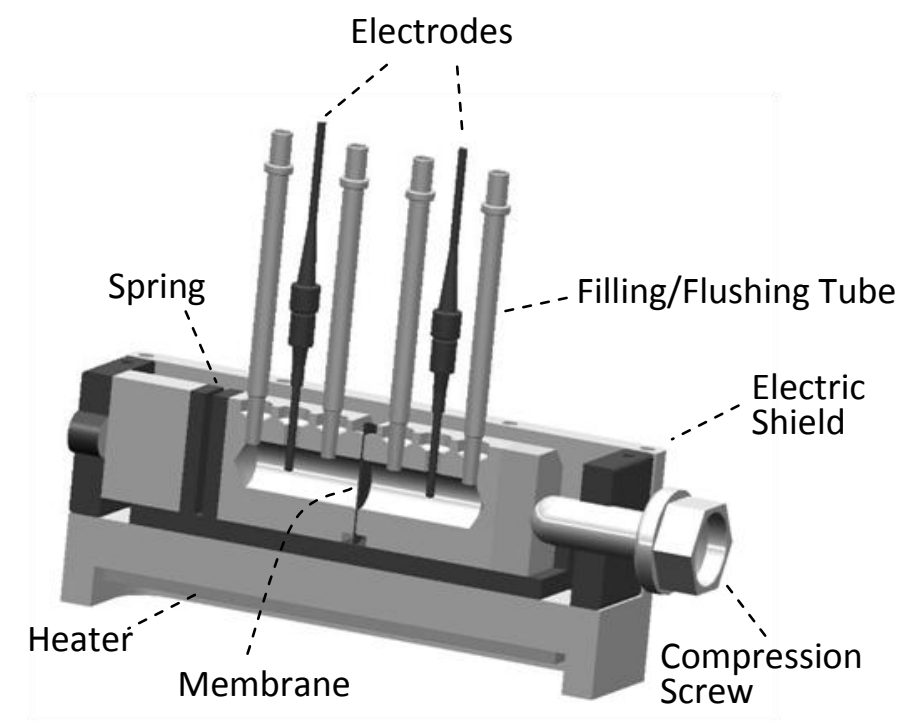

Fig 5 Longitudinal section through conductometric cell ${ }^{9}$ consisting of two cell halves between which the ion track membrane is inserted and compressed by a screw. Four filling/flushing tubes and two electrodes are shown. The cell is heated from the bottom and encased in an electrical shield. The whole assembly is enclosed by a thermal insulation (not shown).

Leak currents suppressed a compression seal. The electrolyte is maintained at a preset temperature and the whole assembly is shielded electrically and thermally by a metallic case. Electrodes and flushing tubes are plugged-in from the top. The growth of the ion track channels is controlled in real-time by electric current measurements down to the sub nA range. To minimize electrode polarization, a sinusoidal voltage with amplitudes below $0.1 \mathrm{~V}$ is used permitting to determine membrane thickness and channel diameter.

\subsection{Track replication}

For electro-replication of the etched ion track channel, the membrane is coated on one side with a metal electrode and inserted into a modified conductometric cell in which the right chamber is replaced by a block of copper serving as cathode. The left chamber is filled with an electrolyte containing the metal or semiconductor ions.

\subsection{Computer interface}

The principle of the data acquisition system is shown in Fig 6. The computer controls the data acquisition card, which converts the digital signal from the computer into an analog voltage, which is applied to the conductometric cell. The output of the conductometric cell is connected with the input of a pico-amperemeter converting current into voltage. Its output voltage is connected via an analog to digital converter to the computer. The current through the etched ion track channel is measured and analyzed using a LabVIEW ${ }^{7}$ based graphical user interface ${ }^{10}$ :

9 http://rsp.eunitt.de : Projects : Controlled Fabrication of Micro Channels : thumbnails : E-Cell 2

10 http://rsp.eunitt.de : Projects : Controlled Fabrication of Micro Channels : thumbnails 
DAQ Card

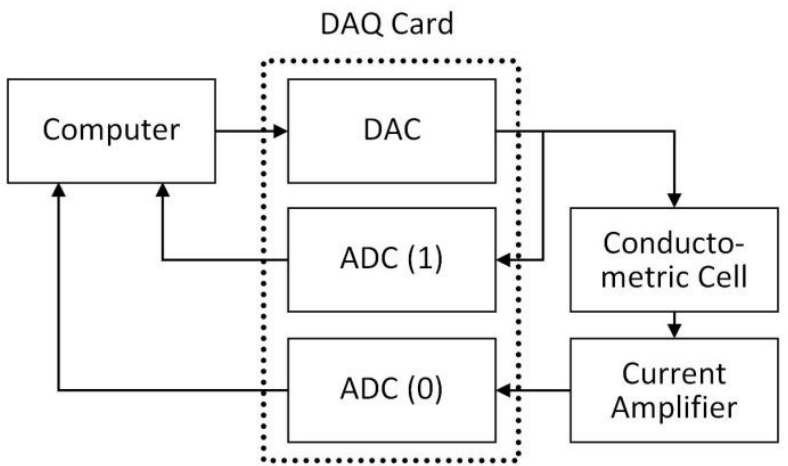

Fig 6 Principle of the data acquisition system ${ }^{11}$ consisting of a computer, a data acquisition card (DAQ Card ${ }^{12}$ ) containing one digital to analog converter (DAC) and two analog to digital converters (ADC), the conductometric cell, and a current amplifier (picoamperemeter).

\subsection{User interface}

One module defines the parameters of the data acquisition board. The next module selects among four modes: The normal operation of the system, the optional use of relays for filling or flushing the electrolyte, the automatic switching between various function generators, and the conditional switching of the applied output voltage. Another module selects among different output function generators with arbitrary shape, amplitude, bias, and frequency. A module exists for calibrating the current amplifier. A module exists for setting the data acquisition parameters, in particular scan rate and time limit of the data acquisition. It provides two acquisition modes: The $d c$ mode acquires current and voltage as a normal time series of data points. The lock-in mode requires a periodic output voltage for determining the phase relation between input voltage and output current. Another module displays the acquired raw data and stores selected data as a new file. It zooms, averages, compresses, and restricts raw data. The selected data are passed to an intermediate storage from which they can be accessed by other modules for further treatment and display. The module can alternatively display all data, only data acquired within a given time interval, all data from a set time onward, and a chart display showing only the most actual data points. The density of the displayed data points is automatically adapted to the screen resolution and can be increased or decreased in steps. Furthermore, a calculation module exists providing a set of subprograms to determine membrane thickness, track etch rate, channel diameter, and wire diameter. In addition, it comprises a conditional option for changing the voltage or switching a relay when reaching a specific threshold. For ion track etching a shape selector exists corresponding to cylindrical, single-cone and double conical shapes. Its output data can be passed to a display module. The module can be used to determine membrane thickness, track etch ratio, channel diameter, and wire length.

\section{Single-ion track applications}

\subsection{Etching of single track}

Real-time controlled etching was applied to single ion track etching (Fig 7). The example demonstrates four steps of extracting results from raw data: break-through time, track etch rate, bulk etch rate, etched track diameter, radius of latent track core (the preferentially etched zone), radius of latent track halo (the cross-linked part of the latent ion track). Experimental parameters: single ion track $\left({ }^{129} \mathrm{Xe}^{27+}, 8.3 \mathrm{MeV} /\right.$ nucleon) in $30 \mu \mathrm{m}$ polycarbonate, etched in $5 \mathrm{M} \mathrm{NaOH}+$ trace of Dowfax 2A1 surfactant at ca. $50{ }^{\circ} \mathrm{C}$. Applied voltage $10 \mathrm{~Hz}$ sine at amplitude $0.1 \mathrm{~V}$.

11 http://rsp.eunitt.de : Projects : Controlled Fabrication of Micro Channels : thumbnails: Data Acquisition

12 National Instruments DAQCard-6062E, http://sine.ni.com/nips/cds/view/p/lang/en/nid/11918 


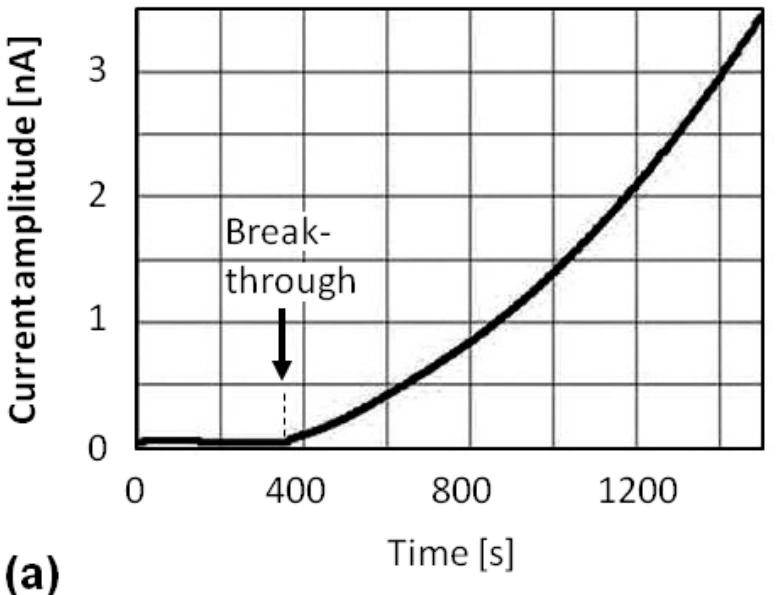

(a)

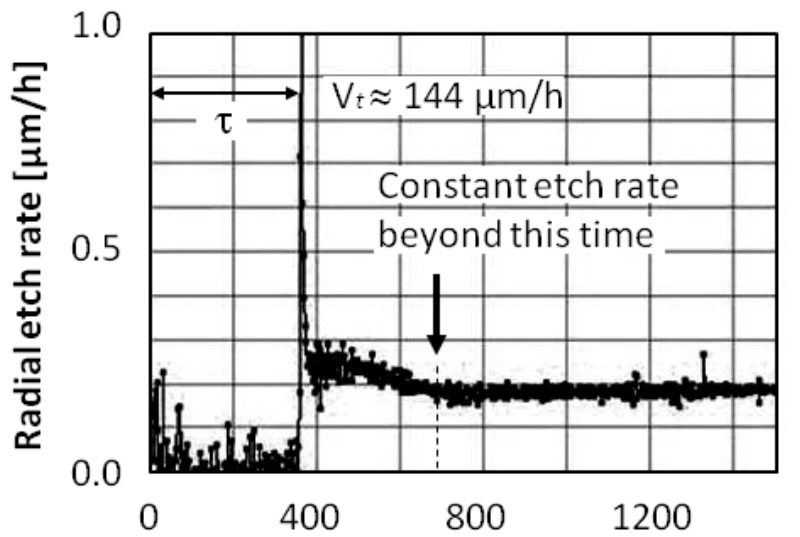

(c)

Time $[\mathrm{s}]$

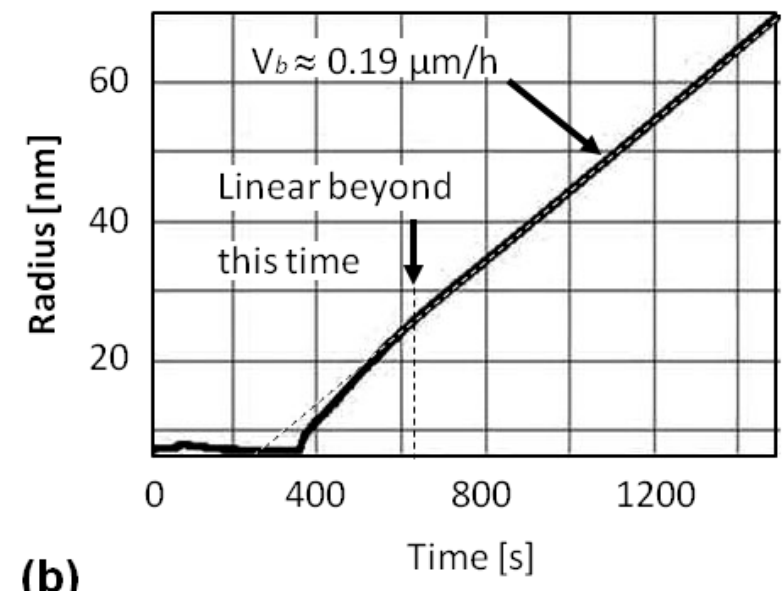

(b)

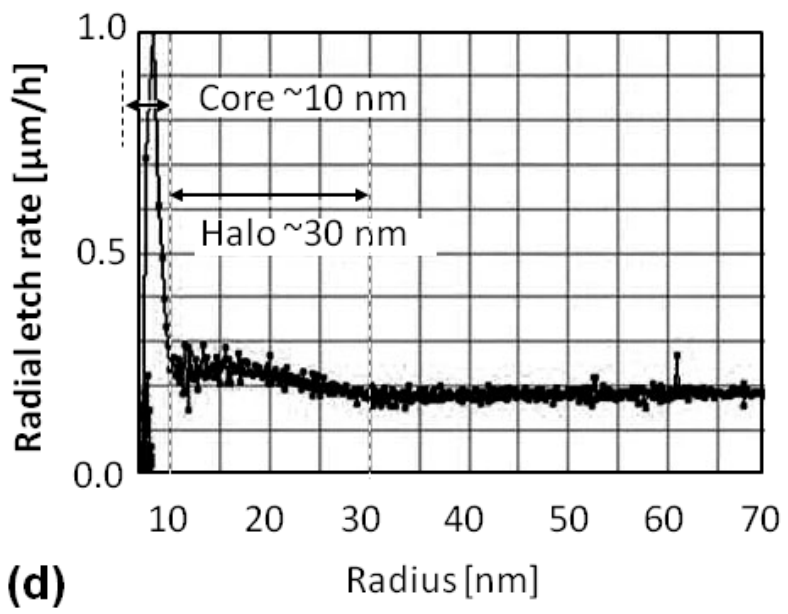

Fig 7 Etching a single-ion track. ${ }^{13}$ (cf. [47-48]) (a) Break-through, followed by a quadratic increase of the electric current through the etched channel. (b) Channel radius calculated from (a). (c) Radial etch rate versus time calculated from (b). (d) Radial etch rate versus radius calculated from (b) and (c). [49]

\subsection{Etching of 100 tracks}

The etching of 100 ion tracks (Fig 8) closely resembles the etching of a single track (Fig 7), however, as expected, with reduced signal to noise ratio.

13 Reprinted from Radiation Measurements, 43, Reimar Spohr, Real-time control of track etching and recent experiments relevant to micro- and nano-fabrication, Figs. 5, 6 Copyright (2008), with permission from Elsevier. 

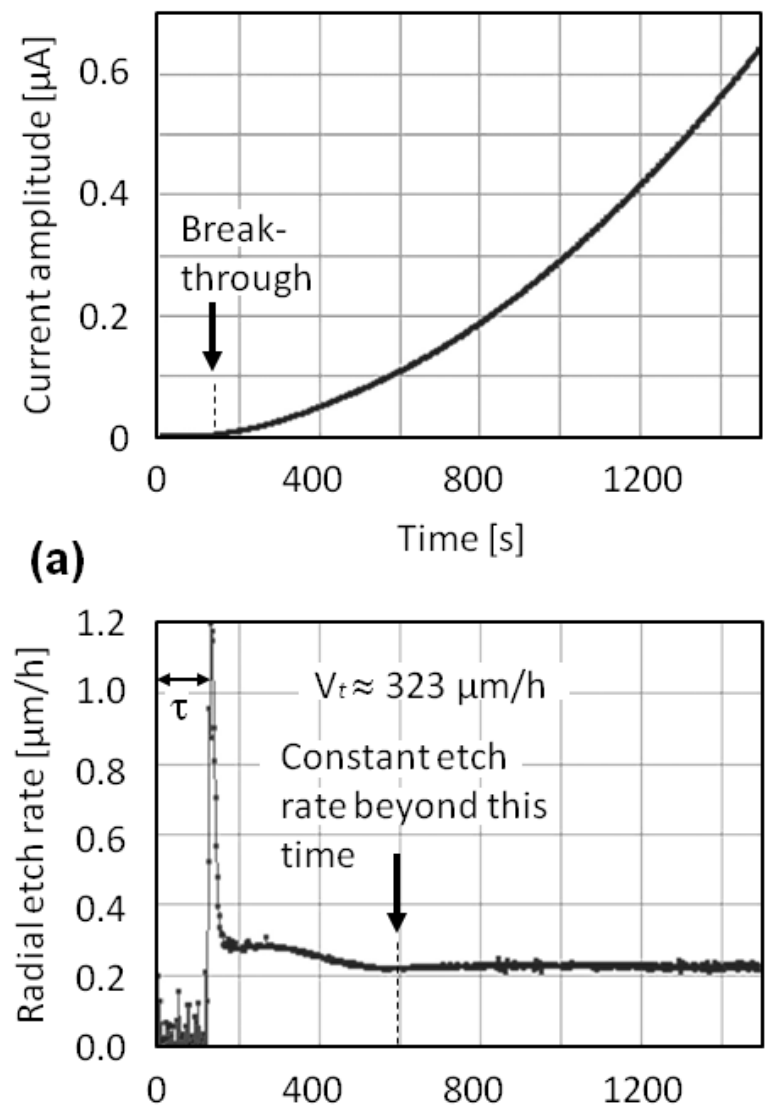

(c)
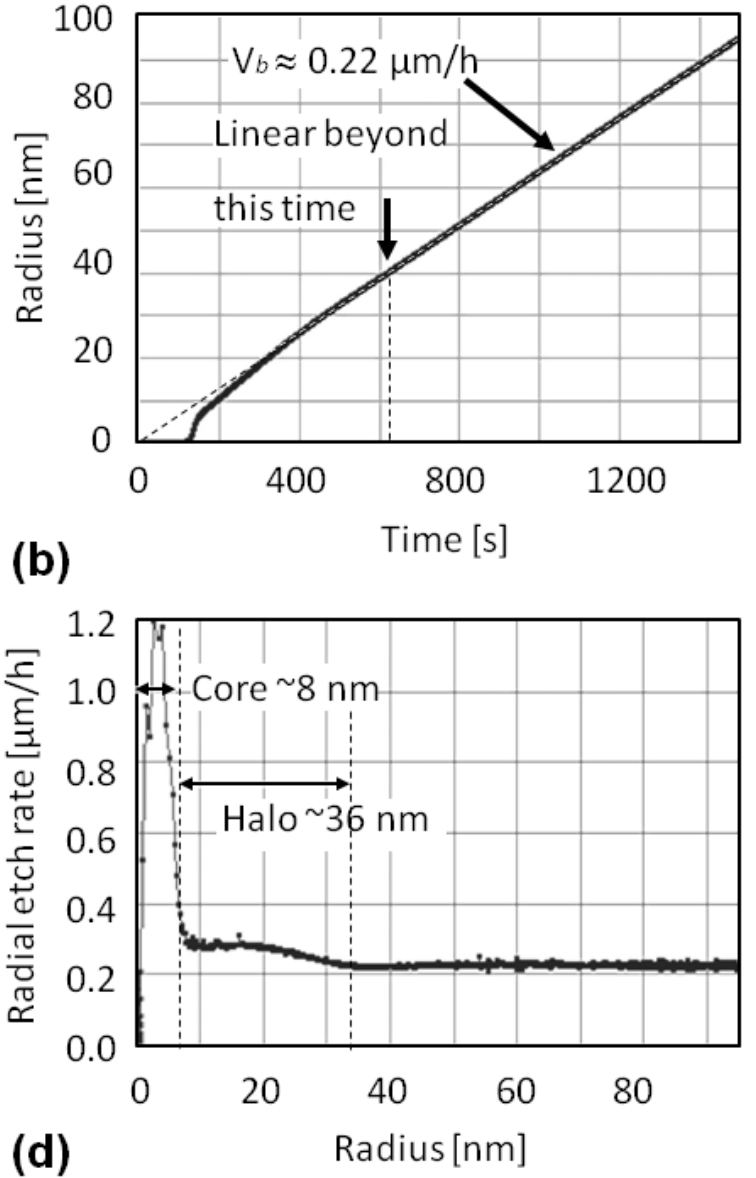

Fig 8 Etching 100 ion tracks $^{14}$ (cf. [47-48]). (a) Break-through, followed by a quadratic increase of the electric current through the etched channel. (b) Channel radius calculated from (a). (c) Radial etch rate versus time calculated from (b). (d) Radial etch rate versus radius calculated from (b) and (c). [49]

\subsection{Probability of double tracks}

Assuming a circular beam spot size of radius $R$ with $N$ etched ion tracks of radius $r$ one obtains a probability for the occurrence of double pores formed by exactly two pores according to

$$
P_{2} \approx 4 P \cdot e^{-4 P}
$$

where $P=N \cdot \pi \cdot r^{2}$ is the nominal porosity. [50] Let us consider the example of 100 ion tracks of $1 \mu \mathrm{m}$ diameter placed at random on a circular area of diameter $100 \mu \mathrm{m}$. Already about 4 double pores will be formed on the average, which is a strong argument for single-pore and focused beam experiments, if double pores are expected to falsify the results.

\subsection{Modifying cone angle}

Ion track etching is governed by the selective removal of the latent ion track by the etch medium as compared to the pristine material. [40] Over short distances, in comparison with the total range of the ion in the solid, the cone half-angle $\alpha$ depends on the track etch ratio, which is defined as the ratio of the track etch rate $v_{\mathrm{t}}$ and the bulk etch rate $v_{\mathrm{g}}$ according to

14 Reprinted from Radiation Measurements, 43, Reimar Spohr, Real-time control of track etching and recent experiments relevant to micro- and nano-fabrication, Figs. 5, 6 Copyright (2008), with permission from Elsevier. 


$$
\alpha=\arcsin \left(\frac{v_{g}}{v_{t}}\right) .
$$

The cone half-angle $\alpha$ can be increased either by increasing $v_{\mathrm{g}}$ or by decreasing $v_{\mathrm{t}}$. In polymers, $v_{\mathrm{g}}$ can be increased by adding organic solvents to the etching solution, in case of PET and polycarbonate (PC) by methanol being added to the basic etchant. On the other hand, the track etch rate $v_{\mathrm{t}}$ can be decreased by thermal annealing before etching is started. This, too, will increase the cone half angle $\alpha$. Conical nanopores are obtained by etching a single ion track membrane from one side only and the asymmetry can be accentuated by retracting the etch-active $\mathrm{OH}^{-}$ions from the etching tip [22].

\subsection{Asymmetric single pores}

In polymers containing oxygen in the backbone of the polymer strand, such as PET and PC, dangling carboxylic groups (R-COOH) are formed on the etched wall and still remain there after flushing off the etch medium. The resulting charged conical nanopores provide a possibility of current rectification. Since the effect depends on $\mathrm{pH}$ it can be used as a basis for sensor development. The current-voltage characteristic can be compared with an electrical diode, however, it rather obeys a power law [34] than an exponential function. The charged groups can be used to attach other groups or biomolecules to the pore walls. But even covalent bonding can be used for attachment if appropriate functional groups exist on the etched surface. This is a starting point for biospecific single-channel sensors [51-60].

\subsection{Surfactant assisted etching}

Cylindrical tracks are obtained if $\alpha$ is very small, i.e. if the track etch rate $v_{\mathrm{t}}$ is large in comparison with the bulk etch rate $v_{\mathrm{g}}$ (Eq. 2). In minerals such as mica track etch ratios above 1000 are possible. For polymers, a special technique was developed at Dubna [61], reducing the surface attack by a monolayer of surfactant molecules unable to penetrate into the etching zone of the ion track (Fig 9). On this basis, using as surfactant alkyldiphenyloxide disulfonate (Dowfax 2A1), the shape of the etched pore channel can be made cylindrical or even cigar-like, as the case requires. The geometry of the pore is governed by the interplay between protection, attack, and diffusion of both etchant and surfactant molecules. Under slow etching conditions, the selectivity of ion track etching was increased, leading to nearly cylindrical channels [62] (Fig 10).
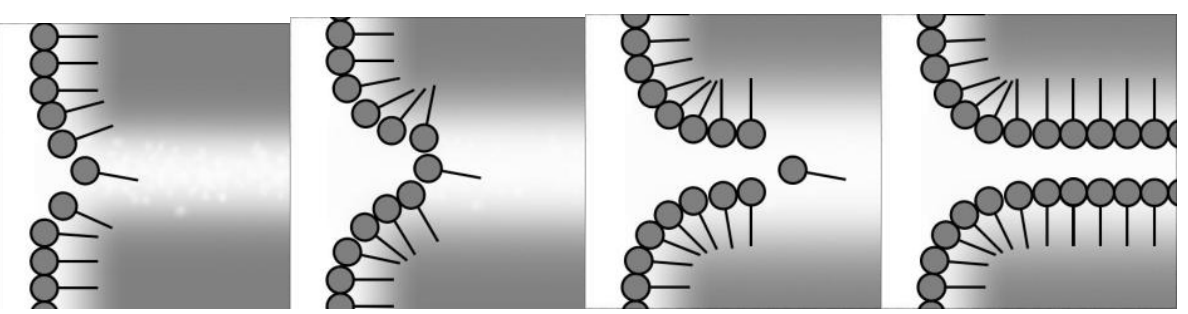

Fig 9 Illustration of surfactant assisted ion track etching. ${ }^{15}$ (From left to right) The diffusion of the surfactant molecules into the etching zone is delayed by their size. At sufficient channel diameter the molecules are able to penetrate into the etching track channel, where they absorb and delay further etching of the track wall. [62] 


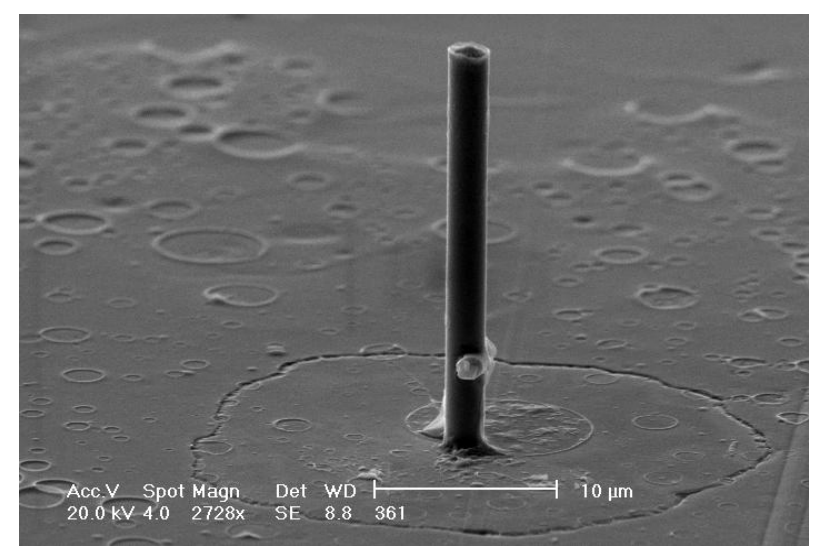

Fig 10 Single wire ${ }^{16}$ [62]; Copper replica of cylindrical ion track channel, electro deposited at $10 \mathrm{nA}$ maximum current. Diameter $2.2 \mu \mathrm{m}$, length $25.6 \mu \mathrm{m}$, bud diameter (visible at bottom) $31 \pm 3 \mu \mathrm{m}$.

\subsection{Magnetoresistance of homogeneous wires}

The magnetoresistance of homogeneous magnetic wires depends strongly on the direction of the applied magnetic field with respect to the wire axis and is termed Anisotropic Magnetic Resistance (AMR), which obtains its maximum if the magnetic field direction is at $90^{\circ}$ with respect to the wire axis. The anisotropic magnetoresistance ratio, AMR ratio, is defined as MR [\%] $=100\left(\mathrm{R}_{\max }-\mathrm{R}_{\min }\right) / \mathrm{R}_{\min }$, where $R_{\max }$ and $R_{\min }$ is the resistance at zero and saturation field, respectively. Fig 11 displays the magnetoresistance curves of a Ni nanowire in a single-channel template with a channel diameter of $(500 \pm 50) \mathrm{nm}$. The AMR curves exhibit the usual dependence on the direction of the applied magnetic field. In the direction parallel to the wire $\left(0^{\circ}\right)$, the effect of the magnetic field on the resistance is very small and the MR ratio is almost zero, while in the direction perpendicular to the wire $\left(90^{\circ}\right)$, the MR effect has a maximum. An AMR ratio of $1.0 \%$ was observed for a Ni nanowire, which is typical of this system. Without magnetic field, the resistance of a Ni nanowire is $198.2 \Omega$. Here, length and diameter of the nanowire are $30 \mu \mathrm{m}$ and $500 \mathrm{~nm}$, respectively. Therefore, the resistivity is estimated to be around 130 $\mathrm{m} \Omega$ confirming that the electrodeposited nanowire has metallic state.

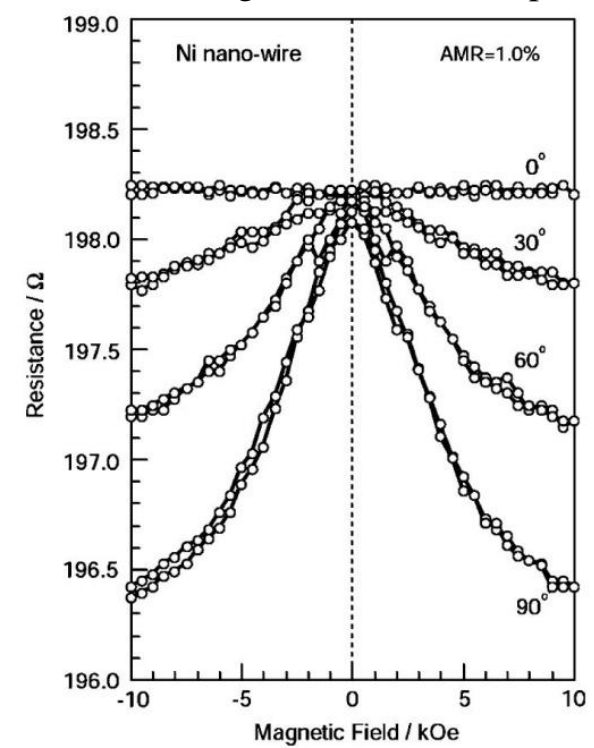

Fig 11 Magnetoresistance of a Ni nanowire ${ }^{17}$ electrodeposited in a single channel with diameter $(500 \pm 50) \mathrm{nm}$.

16 Reprinted from NIM B, 265, n 1, Leo C.T.; Apel, Pavel; Cheung, T.; Westerberg, Lars; Yu, Peter K.N.; Zet, Cristian; Spohr, Reimar; Influence of a surfactant on single ion track etching. Preparing and manipulating individual cylindrical micro wires, p 621-625, Copyright 2007, with permission from Elsevier.

17 With kind permission from Springer Science+Business Media: J Appl Electrochem, 38, 2008, 713-719, T. Ohgai, K. Hjort, R. Spohr, R. Neumann, figure 8-9 


\subsection{Magnetoresistance of layered wires}

Electro-deposition is a technique for selective deposition of metals according to their electronegativity, such as the "standard electrode potential". Pulsed current electro-deposition can be applied to obtain a metallic multilayered structure. If the electrode voltage is switched between two levels, one obtains metal deposits differing on the basis of their electro-negativity. A mixed electrolyte, containing copper as well as cobalt ions, deposits pure copper at low deposition voltage and an alloy of copper and cobalt at high deposition voltage. If the electrolyte contains a large fraction of cobalt, the resulting alloy contains predominantly cobalt and is magnetic. Neighboring magnetic layers can have parallel or antiparallel magnetic orientation. The orientation and electric resistance of the multilayer wire depends on the applied external magnetic field. The magnetic orientation of thin layers can be flipped by spin-polarized electrons from the next layer. This opens a route to spin-dependent devices [63-64].

Periodic potential variations are needed for the fabrication of magnetic/nonmagnetic multilayer structures with anti-parallel spontaneous magnetization. The electric resistance of the wire decreases with increasing magnetic field (Giant Magneto Resistivity, GMR, effect). Thereby a gradual transition from anti-parallel to parallel orientation of neighboring magnetic slabs is induced. Fig 12 displays the angular dependence of the normalized resistance measured at a magnetic field of $10 \mathrm{kOe}$ for a Ni nanowire in a single-channel template with a channel diameter $(500 \pm 50) \mathrm{nm}, \theta$ being the angle between the applied magnetic field and the wire axis (= direction of current). The normalized resistance depends roughly on $\cos 2 \theta$. This result confirms that the track-etched polycarbonate film has straight channels perpendicular to its surface

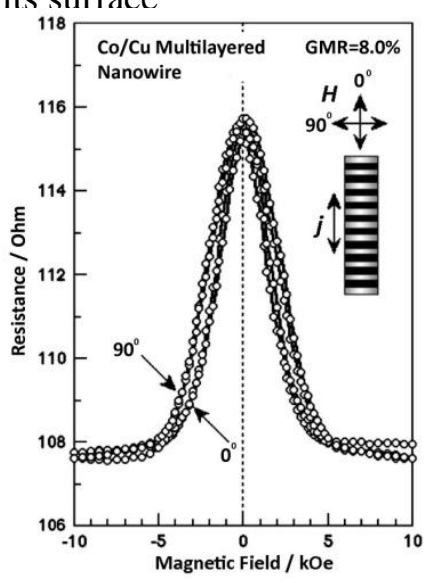

Fig 12 Magnetoresistance of $\mathrm{Co} / \mathrm{Cu}$ multilayered nanowire ${ }^{18}$ with length $30 \mu \mathrm{m}$ electrodeposited in polycarbonate nanochannels. The layer thickness of $\mathrm{Co}$ and $\mathrm{Cu}$ is $10 \mathrm{~nm}$. [27]

\subsection{Relevance of resistance for magnetic sensors}

A systematic study of single wires obtained with the single contact technique developed at EPFL, Lausanne, Switzerland [41] shows, as expected, a linear increase of the resistance with increasing ratio of wire length and wire cross sectional area (Fig 13 a). Surprisingly, the Anisotropic Magneto Resistance (AMR) ratio, characterizing the sensitivity of such sensors, increases with increasing resistance (Fig 13 b).

18 With kind permission from Springer Science+Business Media: J Appl Electrochem, 38, 2008, 713-719, T. Ohgai, K. Hjort, R. Spohr, R. Neumann, figure 8-9 


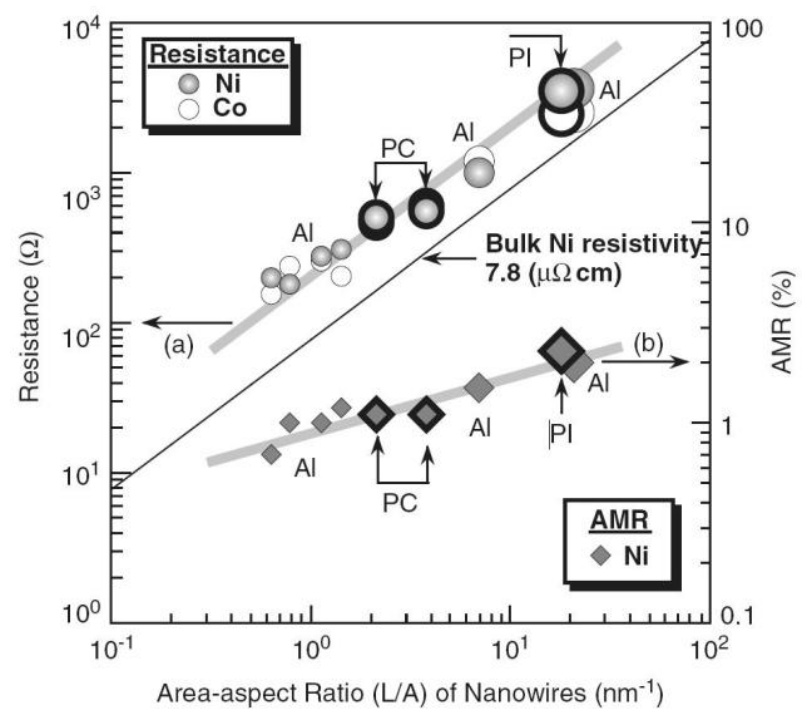

Fig 13 Relation between resistance (a), AMR ratio (b) and area aspect ratio ( $L / A$ : wire length, $L$; cross sectional area, $A$ ) of $\mathrm{Ni}$ and Co nanowires electrodeposited into anodized aluminum (Al), polycarbonate (PC) and polyimide (PI) templates. [44] $]^{19}$

\section{Conclusions and Outlook}

Heavy ion accelerators have paved the way to a widespread use of ion tracks as a precision tool. The technique is capable of generating individual micro- and nanochannels with unprecedented aspect ratios at arbitrary tilt angles. We have described here the possibilities of irradiations with a preset number of ion tracks from some hundred down to one.

On the basis of single-track studies it became possible to control the diameter of etched channels with high precision. The developed techniques can be transferred to random multi-track systems as well as to scanned single-ion microbeams enabling regular pattern of individual objects. In the future such aimed ion microbeams with single-ion detection can be expected to enable unique applications.

Etched ion tracks can be shaped into conical pores with asymmetric current-voltage characteristic, similar to electrical diodes. Their electrical resistance can tell about the $\mathrm{pH}$ of the electrolyte filling the pores. The pores can be rendered biospecific by attaching DNA or enzymes translating the concentration of a substrate molecule into an electrical signal.

Self-assembling surfactant monolayers can be used to control the channel geometry. This concept may enable unexpected new shapes in the future.

Using single ion channels it is possible to fabricate magnetic multilayers with stable electrical contacts. This should promote further studies and the development of devices, such as spin-valves.

Using individual ions, objects down to the nanoscopic scale can be fabricated. They may act as quasi one-dimensional objects if the size of interacting particles becomes comparable to their diameter. On the other hand, wide channels could be used to grow and erode superlattices in "slow motion", closing the technological gap between template synthesis and epitaxial growth.

\section{Acknowledgement}

This report reflects lasting relations between Darmstadt, Dubna, Frankfurt, Iasi, Nagasaki, and Uppsala. The entry of GSI into the field of ion tracks was initiated by late Christoph Schmelzer, promoted by Peter Armbruster, Norbert Angert, and Reinhard Neumann and boosted by Johann Vetter and Christina Trautmann. Göran Thungström contributed a thin window detector for use in single ion irradiations. The crews at the Synchrocyclotron at The Svedberg Laboratory of Uppsala University and at the UNILAC of GSI Darmstadt assisted during experiments and the crew at the Microstructure 
Laboratory (Ångström Laboratory, Uppsala University) in using advanced equipment. Klas Hjort

(Materials Science, Ångström Laboratory, Uppsala University) stimulated the described tape transport system in the frame of the European Research Training Network No HPRN-CT-2000-00047. This work was supported by the Wenner Gren Foundation, Stockholm (Sweden).

\section{References}

[1] R.L. Fleischer, P. B. Price, R.M. Walker; Nuclear tracks in solids: principles and applications; University of California Press, Berkeley, 1975. http://www.ion-tracks.de/nuc/index.html

[2] P.B. Price, G.M. Comstock, R.L. Fleischer, W.R. Giard, H.R. Hart and G.E. Nichols; Cosmic-ray tracks in plastics: The Apollo helmet dosimetry experiment.; Science 172 (1971) 154 .

[3] D.A. Young; Etching of radiation damage in lithium fluoride; Nature 182 (1958) 375-377

[4] R.L. Fleischer, P.B. Price and R.M. Walker; Method of forming fine holes of near atomic dimensions; Rev. Sci. Instr. 34 (1963) 510-512

[5] R.L. Fleischer, P.B. Price and E.M. Symes; Novel filter for biological materials; Science 143 (1964) 249250.

[6] R.W. DeBlois, C.P. Bean; Counting and sizing of submicron particles by the resistive pulse technique; Review of Scientific Instruments 41 (1970) 909-916.

[7] G.E. Possin; A method for forming very small diameter wires; Rev. Sci. Instrum. 41 (1970) 772-774

[8] Early reports on cyclotron irradiations at Dubna performed by V.P. Perelygin and G.N. Flerov date back to around 1970.

[9] P.Yu. Apel, A.Yu. Didyk, V.I. Kuznetsov; Production of single heavy-ion tracks in polymers; Instruments and Experimental Techniques 31 (1988) 1418-1420, ISSN: 0020-4412.

[10] V. I. Kuznetsov, A.Y. Didyk, P.Y. Apel; Production and investigation of nuclear track membranes at JINR; Nucl. Tracks Radiat. Meas. 19 (1991) 919-924.

[11] E. J. van Veldhuizen, C.G. Granqvist, K. Hjort, G. Niklasson, C.G. Ribbing, G. Thornell, L. Nilsson, L. Westerberg, V. Ziemann, J. Thomas; Materials science applications of heavy ion beams; Proceedings of the European Particle Accelerator Conference (EPAC'98, IoP Publishing) (1998) 2460

[12] R. Spohr; Nuclear track irradiations at GSI; Nuclear Tracks 4 (1980) 101-108.

[13] R. Spohr; Method and device to generate a predetermined number of ion tracks; German Patent DE 2951376 C2 (1979).

[14] C. Zet, H. Kiesewetter, M. Skoczylas, L. Westerberg, R. Spohr; A system for irradiating polymer films with a preset number of ions; GSI Scientific Report (2003) 154-154.

[15] C. Zet, C. Fosalau, M. Skoczylas, H. Kiesewetter, R. Spohr; A system for irradiating polymer films with a preset number of ions ; SIELMEN (2003) 167-170.

[16] C. Zet, R. Spohr; FPGA based digital processor for ion irradiation systems; Bulletin of the Polytechnical Institute of Iasi, 52 (2006) 933-942 (ISSN 1223-8139)

[17] H.G. Roggenkamp, H. Kiesewetter, R. Spohr, U. Dauer, L.C. Busch; Production of single pore membranes for the measurement of red blood cell deformability; Biomedizinische Technik 26 (1981) 167-169

[18] B.E. Fischer; The heavy ion microprobe at GSI - used for single ion micromechanics; NIM B 30 (1988) 284288

[19] B.E. Fischer, M. Heiss; A facility to mass-produce ordered single-hit patterns in continuous $35 \mathrm{~mm}$ films ; Nuclear Instruments and Methods in Physics Research Section B 260 (2007) 442-444

[20] J.P. Pekola, J.C. Davis, Zhu Yu-Qun, R.N.R. Spohr, P.B. Price, R.E. Packard; Suppression of the critical current and the superfluid transition temperature of $3 \mathrm{He}$ in a single submicon cylindrical channel; Journal of Low Temperature Physics, 67 (1987) 47-63.

[21] C. Trautmann, R. Spohr, J. Vetter, G. Eska, Y. Hirayoshi; Search for superfluid Josephson effect; Nucl. Tracks Radiat. Meas., 19 (1991) 967-970.

[22] P.Y. Apel, Y.E. Korchev, Z. Siwy, R. Spohr, M. Yoshida; Diode-like single-ion track membrane prepared by electro-stopping; Nucl. Instrum. Methods Phys. Res., Sect. B 184 (2001) 337-346.

[23] Z. Siwy, P. Apel, D. Dobrev, R. Neumann, R. Spohr, C. Trautmann, K. Voss; Ion transport through asymmetric nanopores prepared by ion track etching; Nuclear Instruments \& Methods in Physics Research, Section B: Beam Interactions with Materials and Atoms 208 (2003) 143-148. 
[24] T. Ohgai, L. Gravier, X. Hoffer, M. Lindeberg, K. Hjort, R. Spohr, J.P. Ansermet; Template synthesis and magnetoresistance property of $\mathrm{Ni}$ and $\mathrm{Co}$ single nanowires electrodeposited into nanopores with a wide range of aspect ratios; Journal of Physics D (Applied Physics) 36 (2003) 3109-3114.

[25] T. Ohgai, I. Enculescu, C. Zet, L. Westerberg, K. Hjort, R. Spohr, R. Neumann; Magneto-sensitive nickel nanowires fabricated by electrodeposition into multi- and single-ion track templates. Journal of Applied Electrochemistry 36 (2006) 1157-1162

[26] I. Enculescu, M.E. Toimil-Molares, C. Zet, M. Daub, L. Westerberg, R. Neumann, R. Spohr; Current perpendicular to plane single-nanowire GMR sensor; Applied Physics A (Materials Science Processing) A 86 (2007) 43-47.

[27] T. Ohgai, K. Hjort, R. Spohr, R. Neumann; Electrodeposition of cobalt based ferro-magnetic metal nanowires in polycarbonate films with cylindrical nanochannels fabricated by heavy-ion-track etching; J Appl Electrochem 38 (2008) 713-719.

[28] http://ion-tracks.de : Picture gallery : Track etching

[29] K. Healy; Nanopore-based single-molecule DNA analysis. Nanomedicine 2 (2007) 459-481.

[30] E.A. Heins, Z.S. Siwy, L.A. Baker, C.R. Martin; Detecting single porphyrin molecules in a conically shaped synthetic nanopore. Nano Letters 5 (2005) 1824-1829.

[31] K. Healy, B. Schiedt, A.P. Morrison; Solid-State nanopore technologies for nanopore based DNA analysis. Nanomedicine 2 (2007) 875-897

[32] C. Dekker; Solid-state nanopores. Nature Nanotechnology 2 (2007) 209-215

[33] M. Ali, B. Schiedt, K. Healy, R. Neumann, W. Ensinger; Modifying the surface charge of single track-etched conical nanopores in polyimide. Nanotechnology 19 (2008) 085713-085719

[34] Z. Siwy; Ion-current rectification in nanopores and nanotubes with broken symmetry. Adv. Function. Mat. 16 (2006) 735-746.

[35] Y. Choi, L.A. Baker, H. Hillebrenner, C.R. Martin; Biosensing with conically shaped nanopores and nanotubes. Phys. Chem. Chem. Phys. 8 (2006) 4976-4988.

[36] R. Schoch, J. Han, P. Renaud; Transport phenomena in nanofluidics. Rev. Mod. Phys. 80 (2008) 839-883

[37] C.R. Martin; Nanomaterials: a membrane-based synthetic approach. Science, 266 (1994) 1961

[38] T. Schenkel, V. Radmilovic, E.A. Stach, S.-J. Park, A. Persaud; Formation of a few nanometer wide holes in membranes with a dual beam focused ion beam system; Journal of Vacuum Science \& Technology B 21 (2003) 2720-2723

[39] D.J. Stokes, L. Roussel, O. Wilhelmi, L.A. Giannuzzi, D.H.W. Hubert; Recent advances in FIB technology for nano-prototyping and nano-characterisation. Ion-Beam-Based Nanofabrication Symposium (2007) 15-20

[40] R. Spohr; Status of ion track technology - prospects of single tracks; Radiation Measurements 40 (2005) 191-202

[41] A. Fabian, C. Terrier, S.S. Guisan, X. Hoffer, M. Dubey, L. Gravier, J.P. Ansermet, J.E. Wegrowe; Currentinduced two-level fluctuations in pseudo-spin-valve $(\mathrm{Co} / \mathrm{Cu} / \mathrm{Co})$ nanostructures. ; Physical Review Letters 91 (2003) 257209

[42] N. Fertig, C. Meyer, R.H. Blick, C. Trautmann, J.C. Behrends; Microstructured glass chip for ion-channel electrophysiology; Phys. Rev. E 64 (2001) 040901-040904.

[43] N. Fertig, R.H. Blick, J.C. Behrends; Whole cell patch clamp recording performed on a planar glass chip; Biophysical Journal 82 (2002) 3056-3062.

[44] T. Ohgai, L. Gravier, X. Hoffer, M. Lindeberg, K. Hjort, R. Spohr, J.P. Ansermet; Template synthesis and magnetoresistance property of $\mathrm{Ni}$ and $\mathrm{Co}$ single nanowires electrodeposited into nanopores with a wide range of aspect ratios; J. Phys. D: Appl. Phys. 36 (2003) 3109-3114

[45] G.G. Thungström, L. Westerberg, R. Spohr, C.S. Petersson; Fabrication and characterization of thin $\Delta \mathrm{E}$ detectors for spectroscopic application; Nuclear Instruments \& Methods in Physics Research, Section A (Accelerators, Spectrometers, Detectors and Associated Equipment) 546 (2005) 312-318.

[46] R. Spohr, P.Y. Apel, H. Kiesewetter, M. Skoczylas, C. Zet; Elektrolytische Zellenanordnung; German Patent Office Munich DE102005020734B3, issued 19.10.2006.

[47] P.Y. Apel, G. Pretzsch; Investigation of the radial pore-etching rate in a plastic track detector as a function of the local damage density around the ion path; Nuclear Tracks and Radiation Measurements 11 (1986) 45-53.

[48] L. I. Samoilova, P.Y. Apel; Etching of small pores in PETP by different alkalis ; Radiation Measurements 25 (1995) 717-720. 
[49] R. Spohr; Real-time control of track etching and recent experiments relevant to micro and nano fabrication. Radiat. Meas. 43 (2008) 560-570.

[50] C. Riedel, R. Spohr; Statistical Properties of Etched Nuclear Tracks. I. Analytical theory and computer simulation; Radiation Effects 42 (1979) 69-75

[51] J.D. Uram, K. Ke, A.J. Hunt, M. Mayer; Label-free affinity assays by rapid detection of immune complexes in submicrometer pores. Angewandte Chemie, 45 (2006) 2281-2285

[52] Z. Siwy, L. Trofin, P. Kohli, L.A. Baker, C. Trautmann, C.R. Martin; Protein biosensors based on biofunctionalized conical gold nanotubes; Journal of the American Chemical Society 127 (2005) 5000-5001

[53] M. Ali, B. Schiedt, R. Neumann, W. Ensinger; Biosensing with functionalized single asymmetric polymer nanochannels; Submitted to Macromol Biosci. 2009 Aug 14. (Epub ahead of print).

[54] H. Bayley, C.R. Martin; Resistive-pulse sensing - from microbes to molecules; Chemical Reviews 100 (2000) 2575-2594.

[55] L.T. Sexton, L.P. Horne, S.A. Shernill, G.W. Bishop, L.A. Baker, C.R. Martin; Resistive-pulse studies of proteins and protein antibody complexes using a conical nanotube sensor. JACS 129 (2007) 1314713152

[56] J.H. Wang, C.R. Martin; A new drug-sensing paradigm based on ion-current rectification in a conically shaped nanopore. Nanomedicine 3 (2008) 13-20

[57] M. Ali, B. Yameen, R. Neumann, W. Ensinger, W. Knoll, O. Azzaroni; Biosensing and supramolecular bioconjugation in single conical polymer nanochannels. Facile incorporation of biorecognition elements into nanoconfined geometries. JACS 130 (2008) 16351-16357

[58] C.C. Harrell, Z.S. Siwy, C.R. Martin; Conical nanopore membranes: controlling the nanopore shape; Small 2 (2006) 194-198

[59] L.T. Sexton, L.P. Horne, C.R. Martin; Developing synthetic conical nanopores for biosensing applications

[60] M. Ali, P. Ramirez, S. Mafe, R. Neumann, W. Ensinger; A pH-tunable nanofluidic diode with a broad range of rectifying properties; Nano 3 (2009) 603-608

[61] P.Y. Apel, I. V. Blonskaya, A.Y. Didyk, S.N. Dmitriev, O.L. Orelovitch, D. Root, L.I. Samoilova, V.A. Vutsadakis; Surfactant-enhanced control of track-etch pore morphology; Nucl. Instrum. Methods Phys. Res., Sect. B 179 (2001) 55-62

[62] L.C.T. Man, P.Y. Apel, T. Cheung, L. Westerberg, P.K.N. Yu, C. Zet, R. Spohr; Influence of a surfactant on single ion track etching. Preparing and manipulating individual cylindrical micro wires; Nucl. Instrum. Methods Phys. Res., Sect. B 265 (2007) 621-625.

[63] B. Doudin, J.P. Ansermet; Nanostructuring materials for spin electronics; Europhys. News 28 (1997) 14-17.

[64] D. Kelly, J.E. Wegrowe, Trong-kha Truong, X. Hoffer, J.P. Ansermet; Spin-polarized current-induced magnetization reversal in single nanowires ; Phys. Rev. B 68 (2003) 134425. 


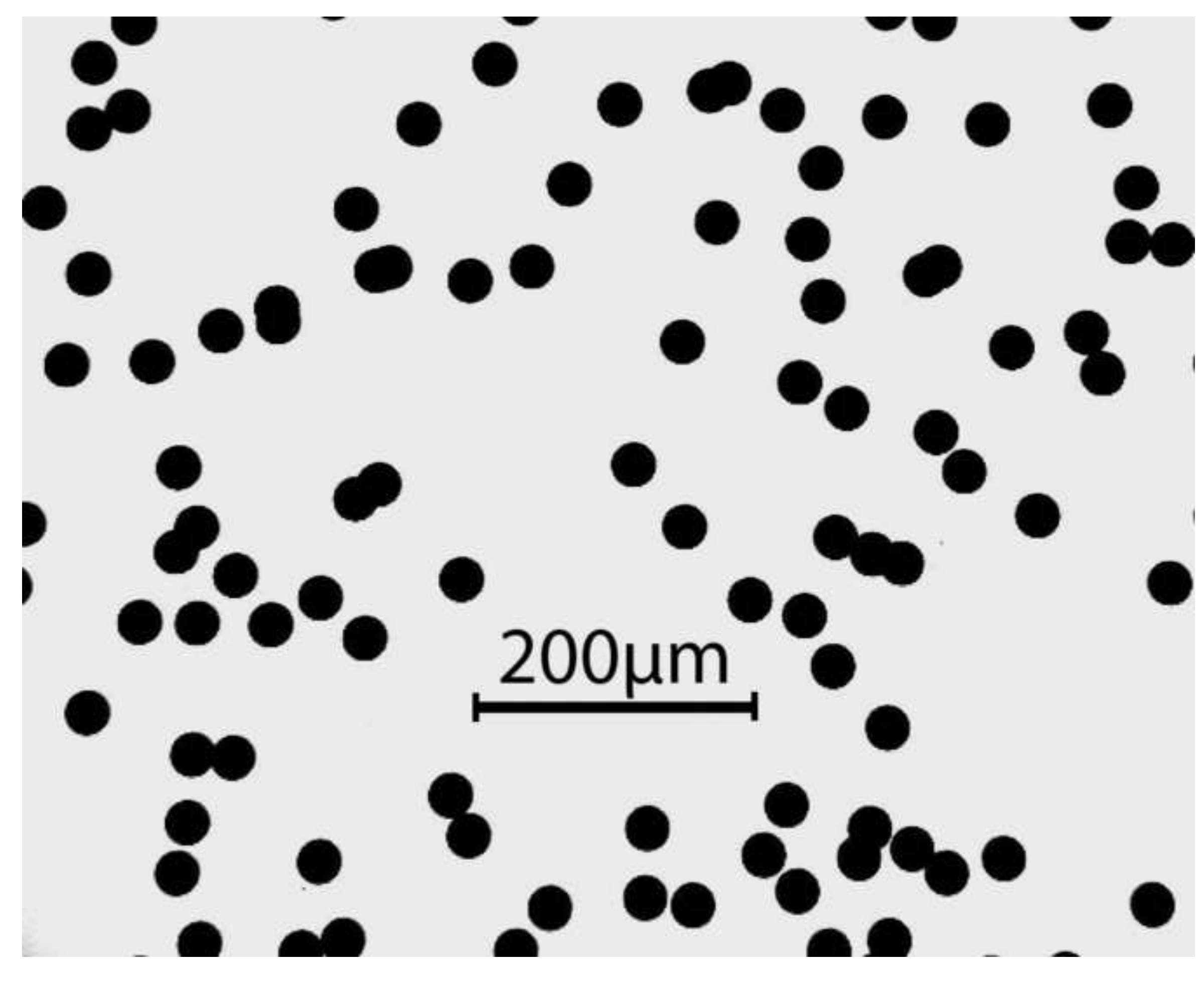



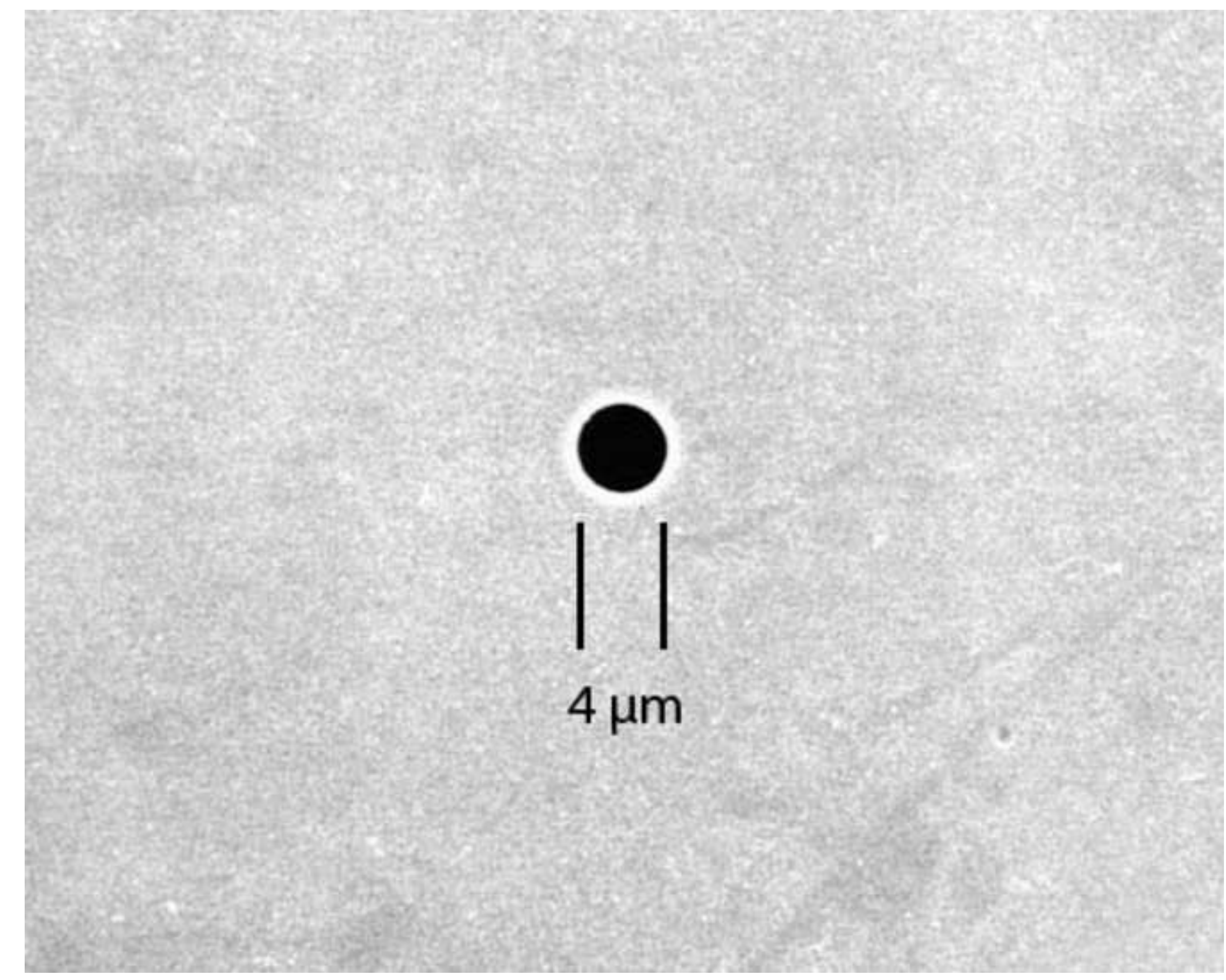


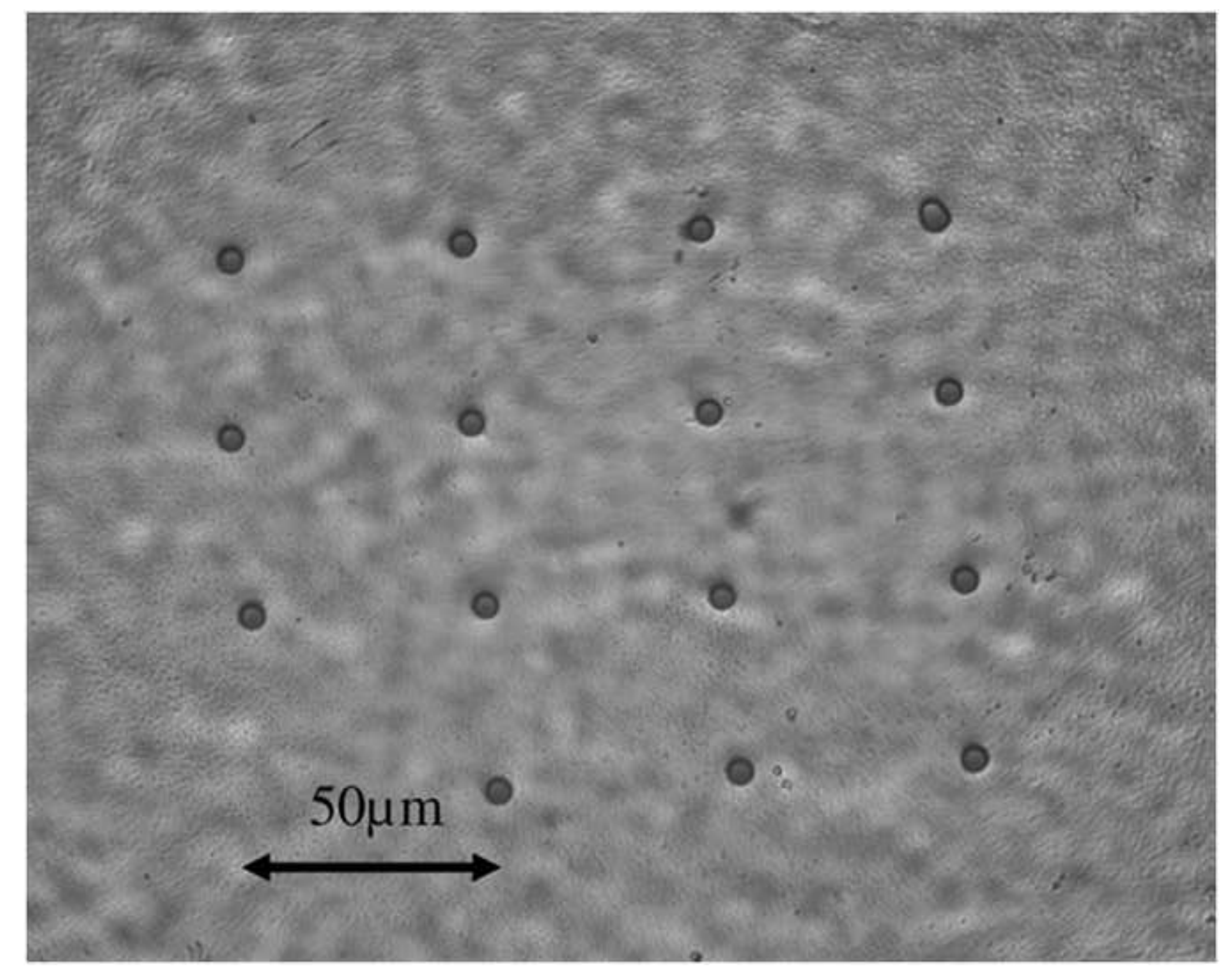

Figure 1-:

\section{$50 \mu \mathrm{m}$}

กิ.

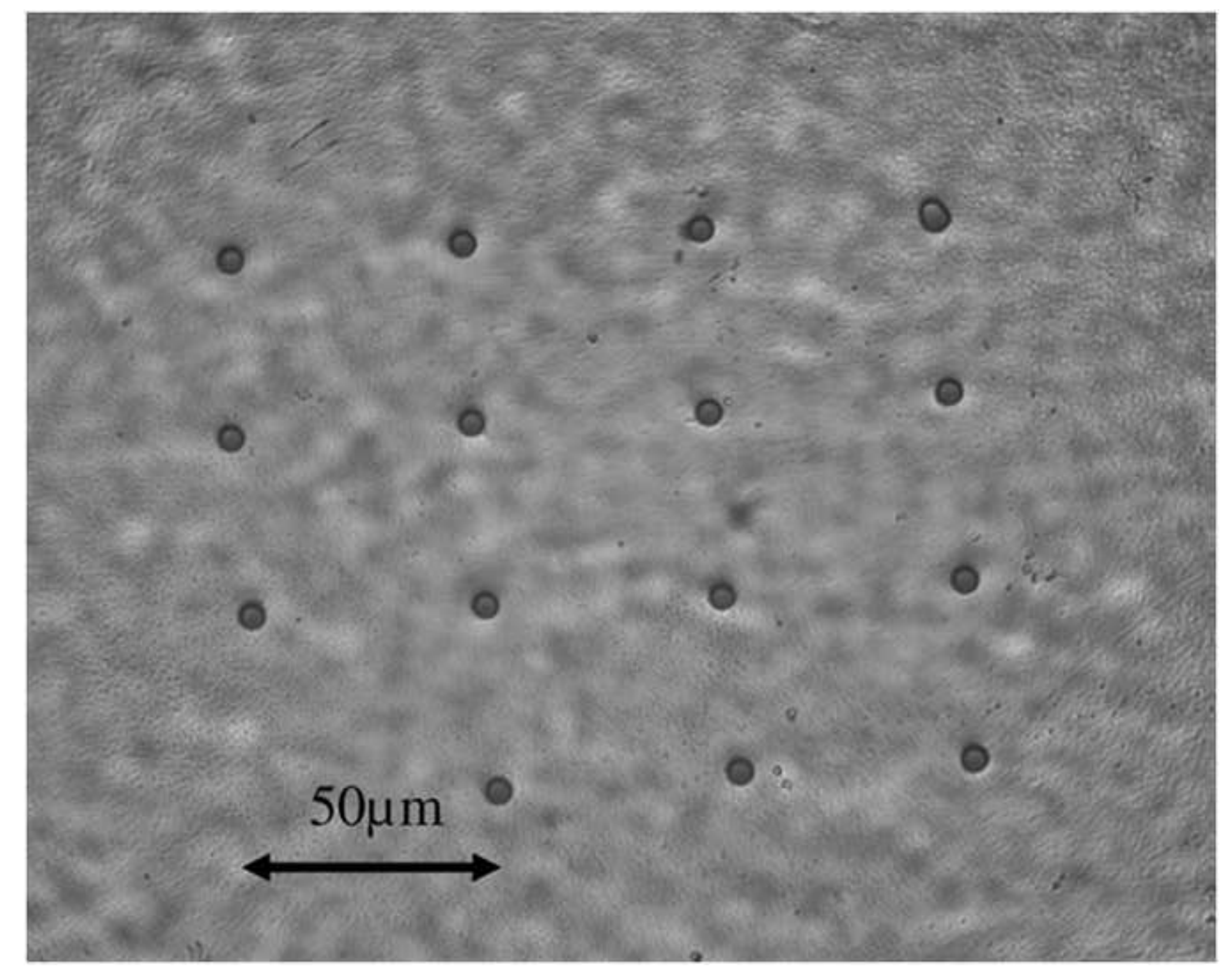




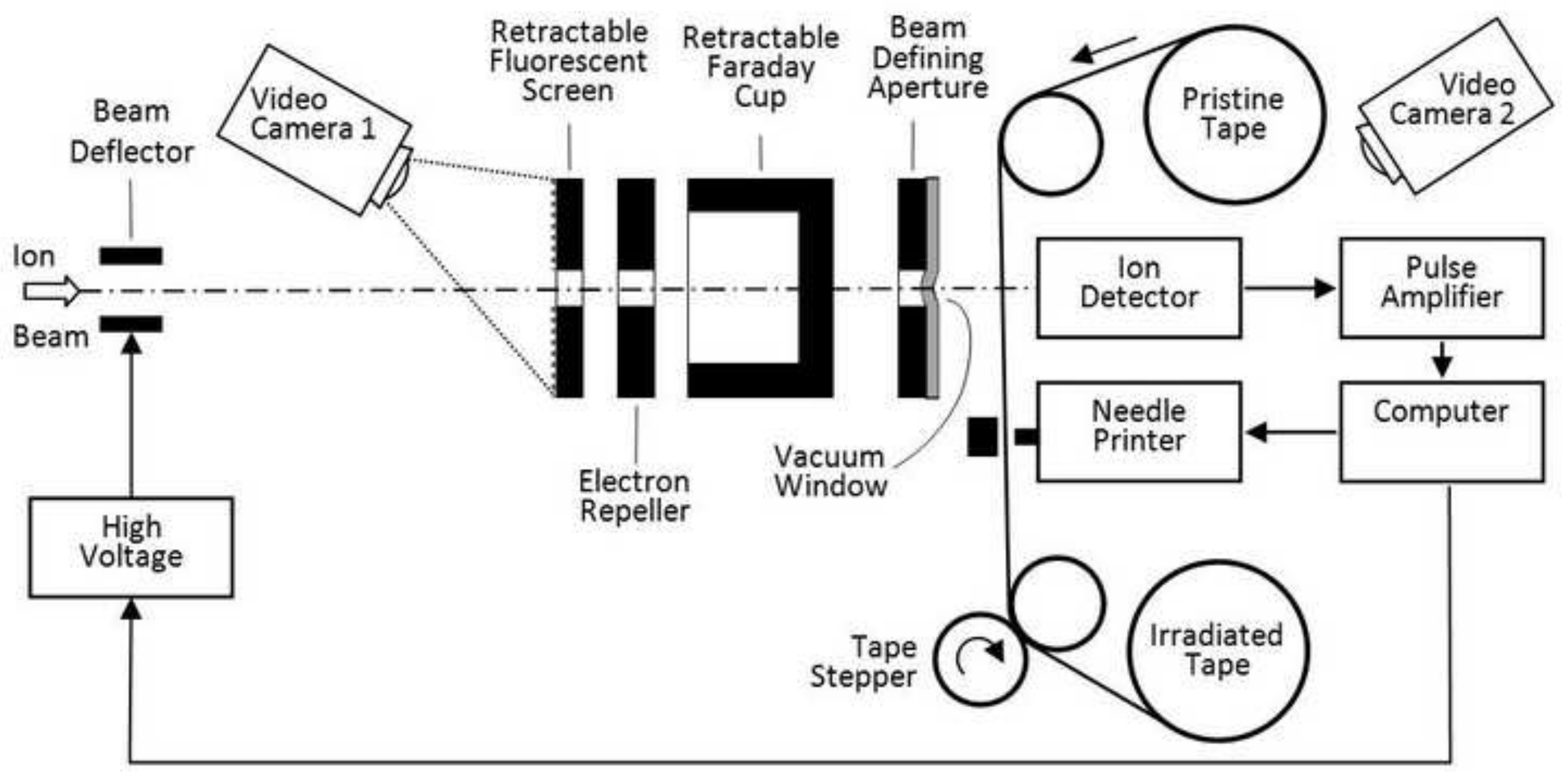




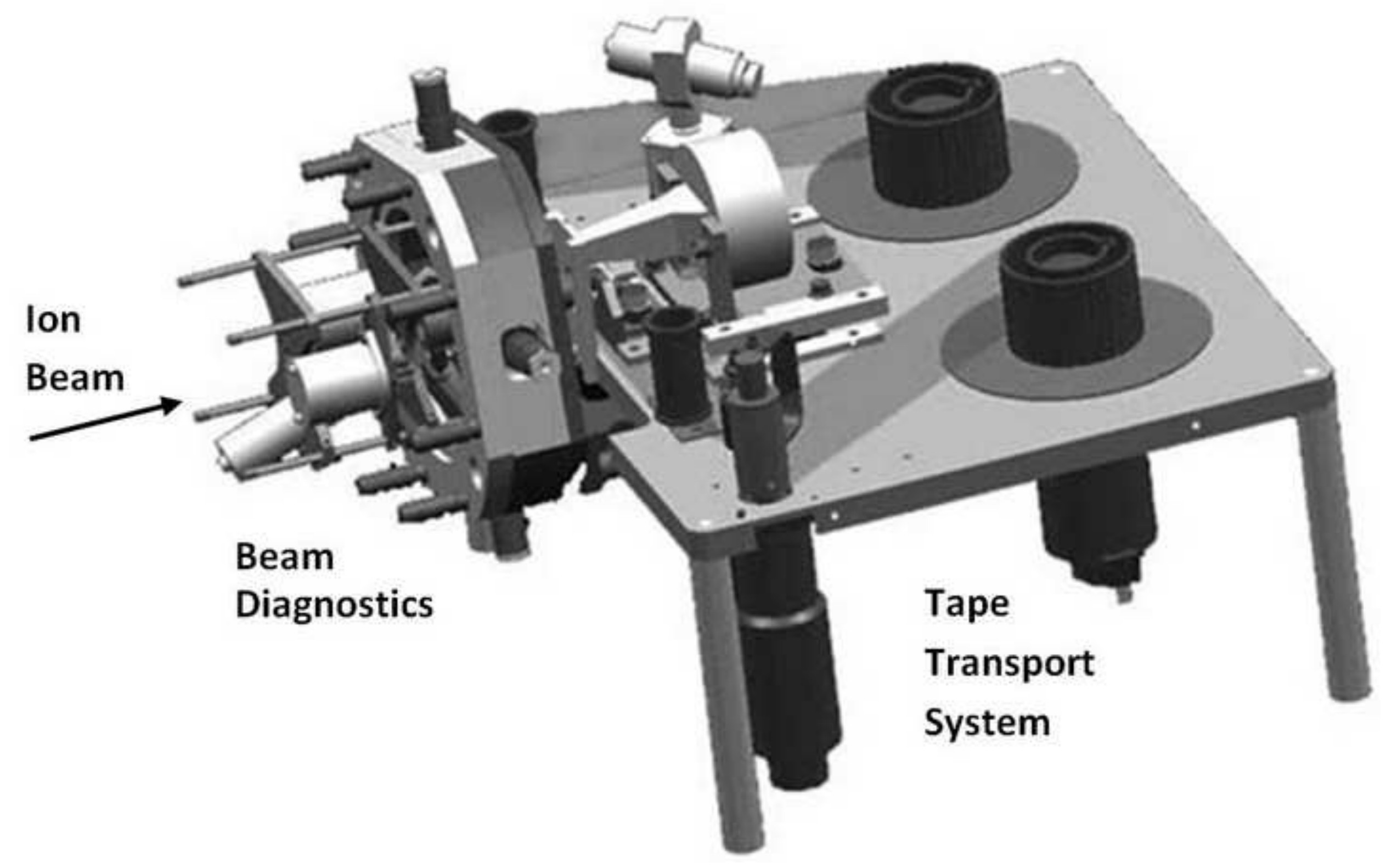

Diagnostics

Transport

Ion

Beam

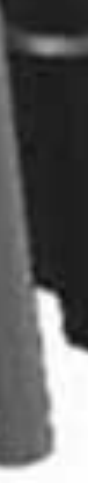

\section{System \\ Tape}

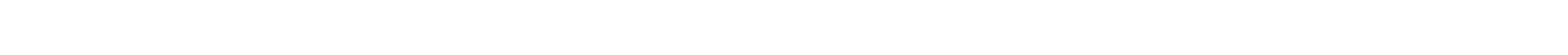




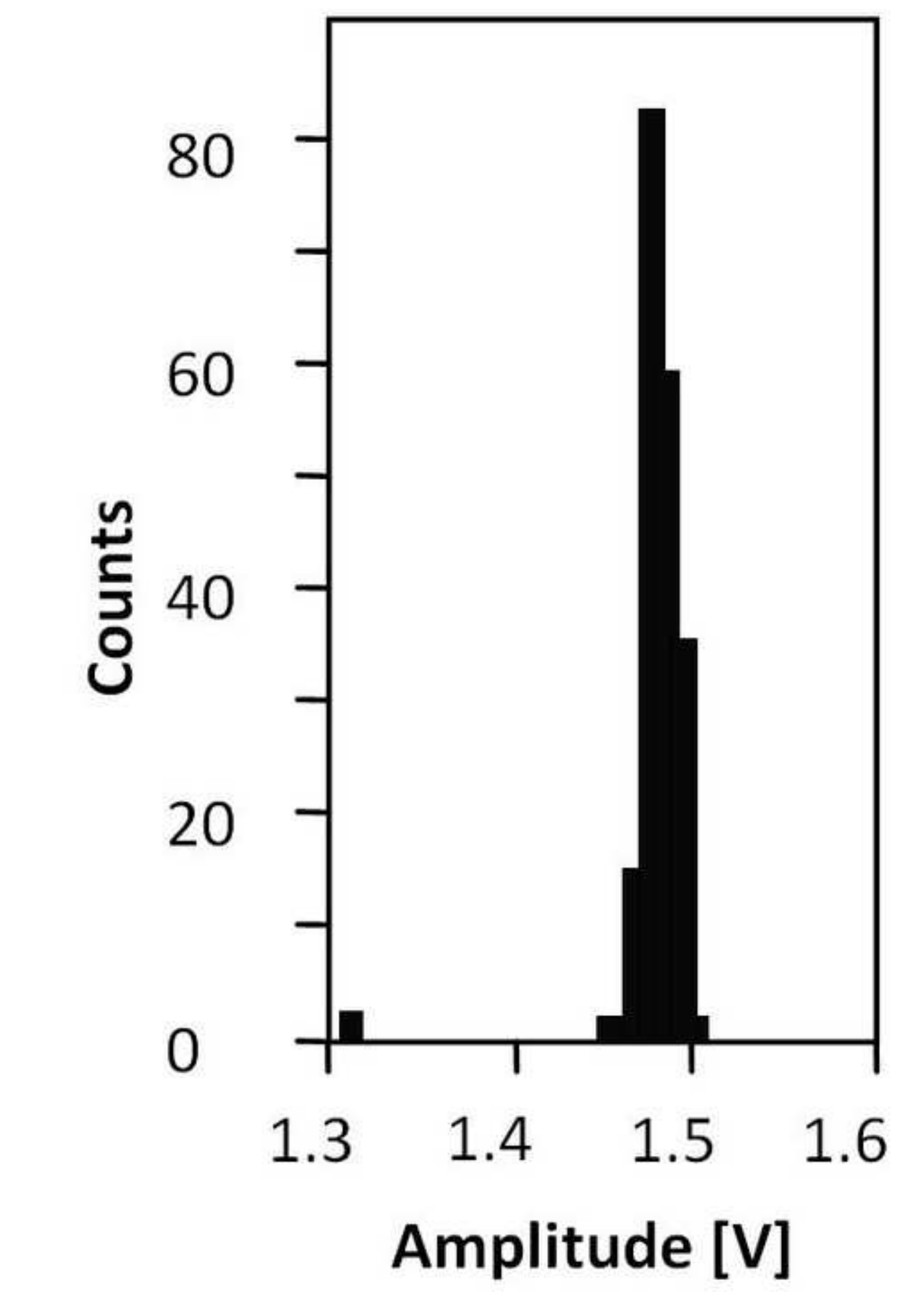

Figure 4

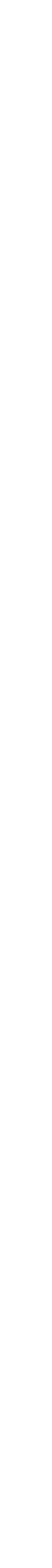

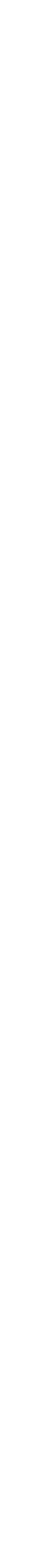

-

年

列
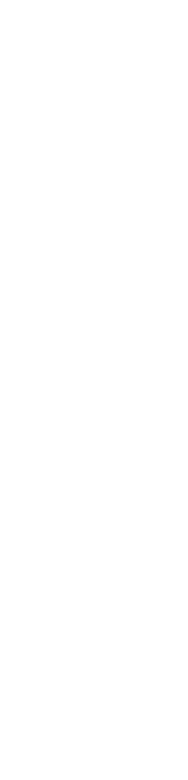

. 


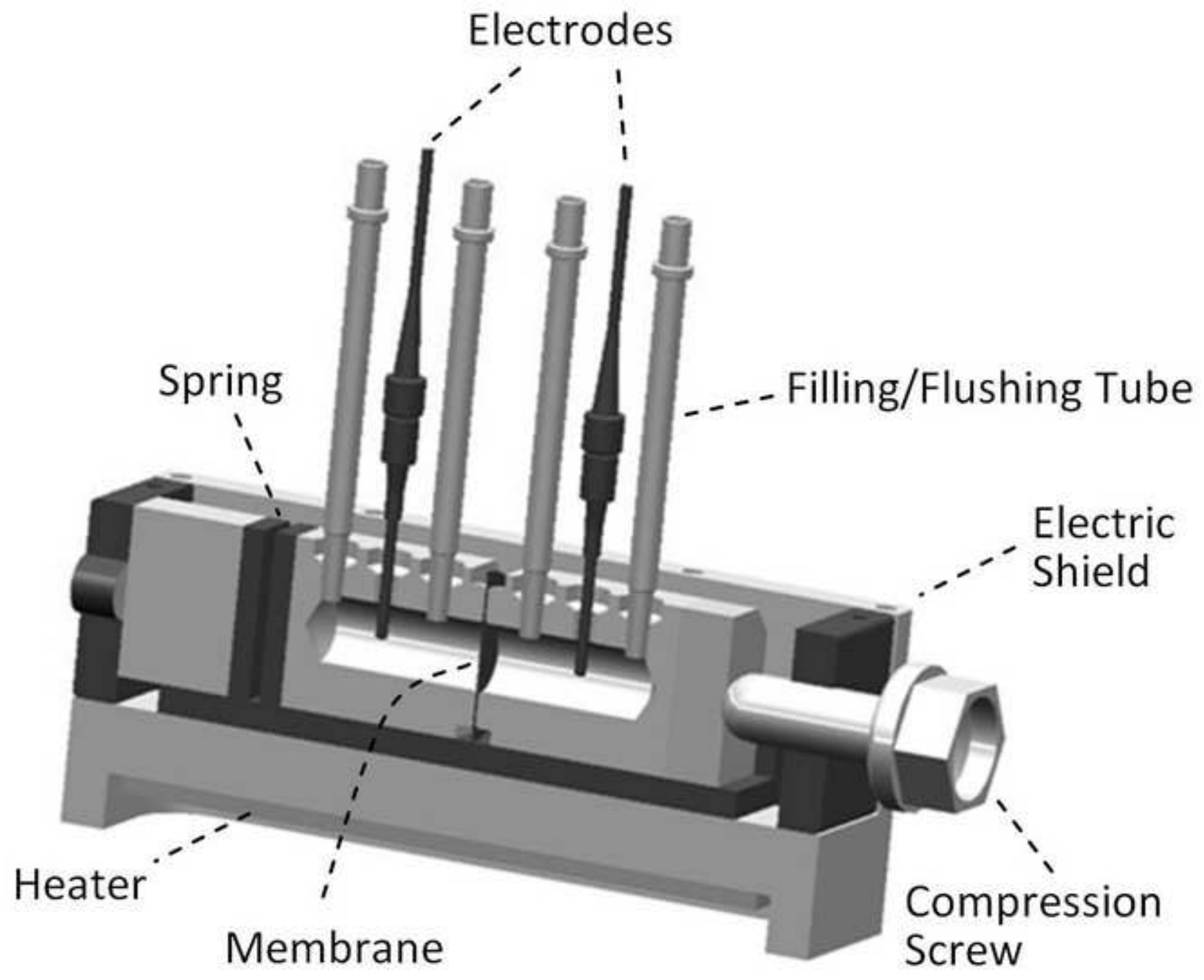

\section{Electrodes}




\section{DAQ Card}

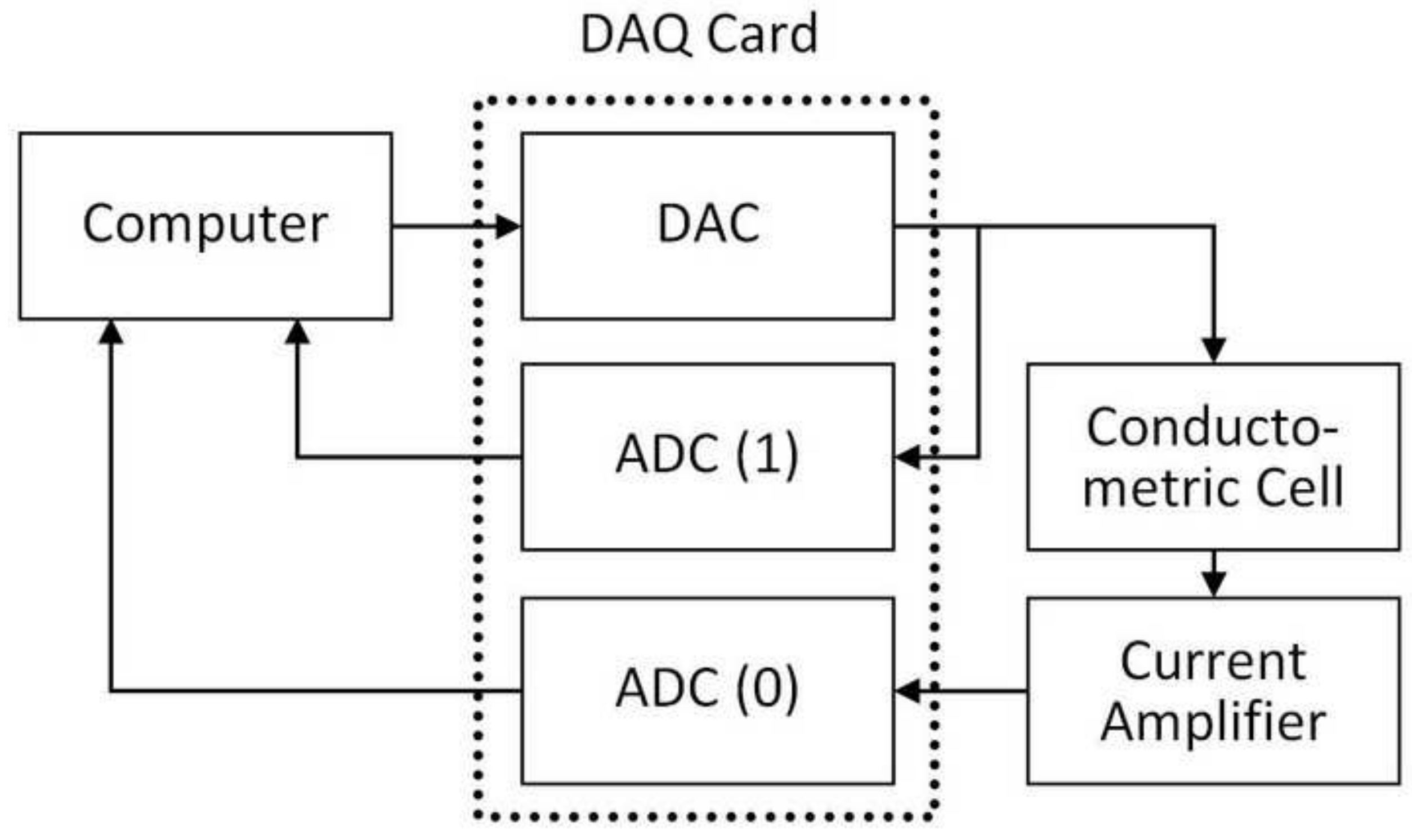




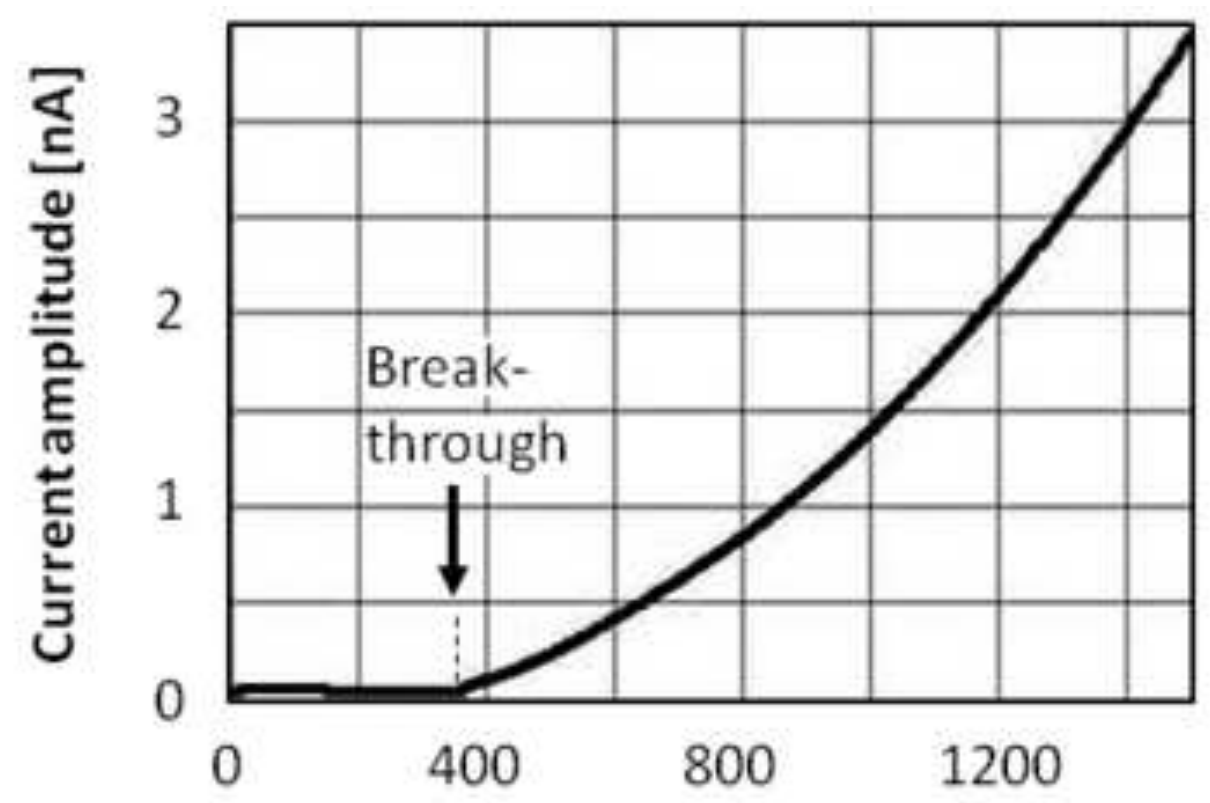

(a)

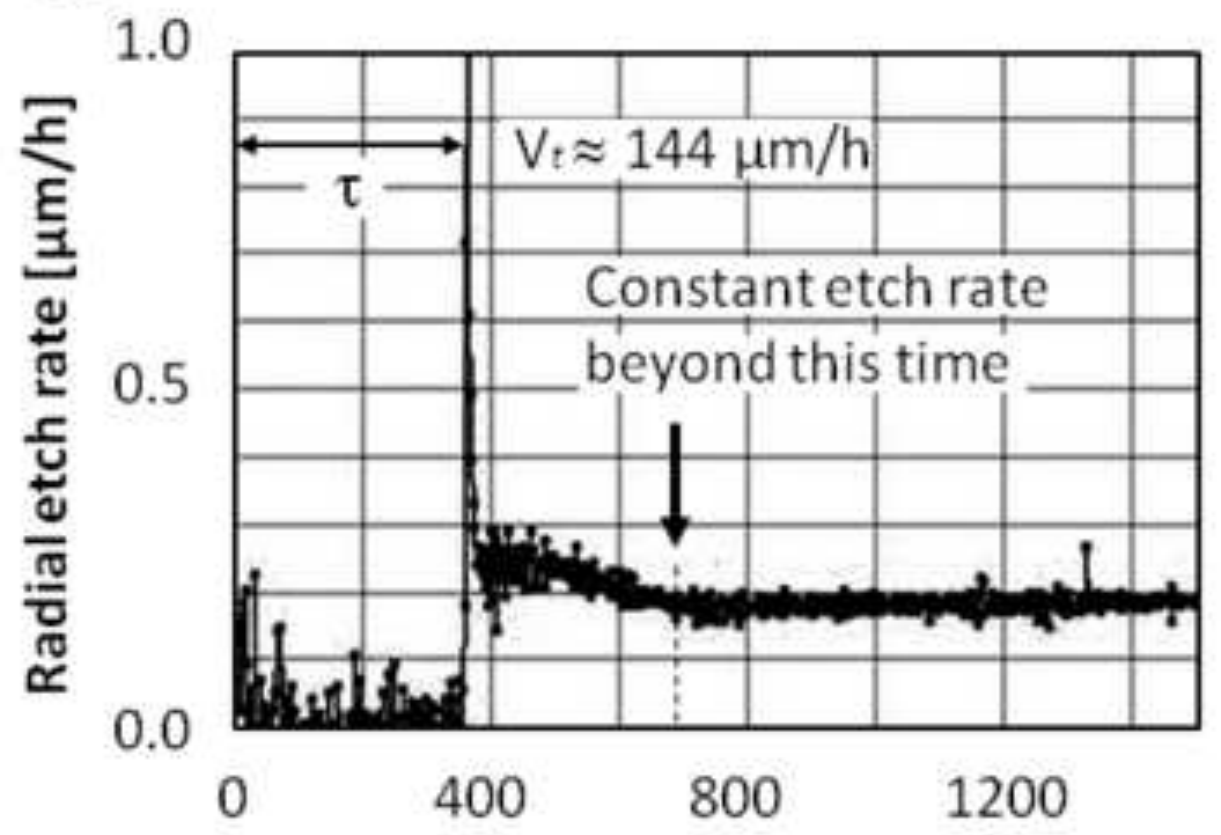

(c)
Time [s]

(b)

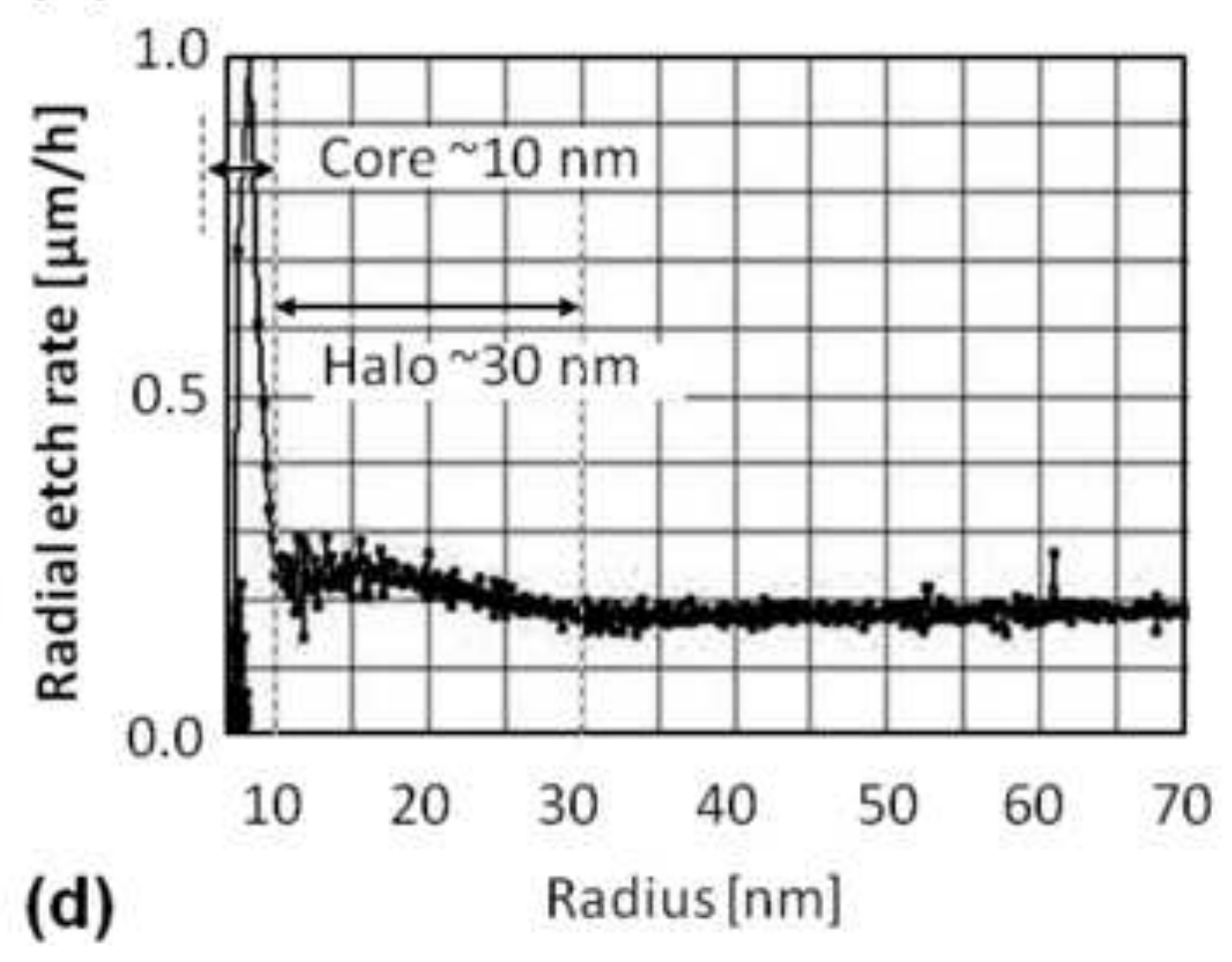

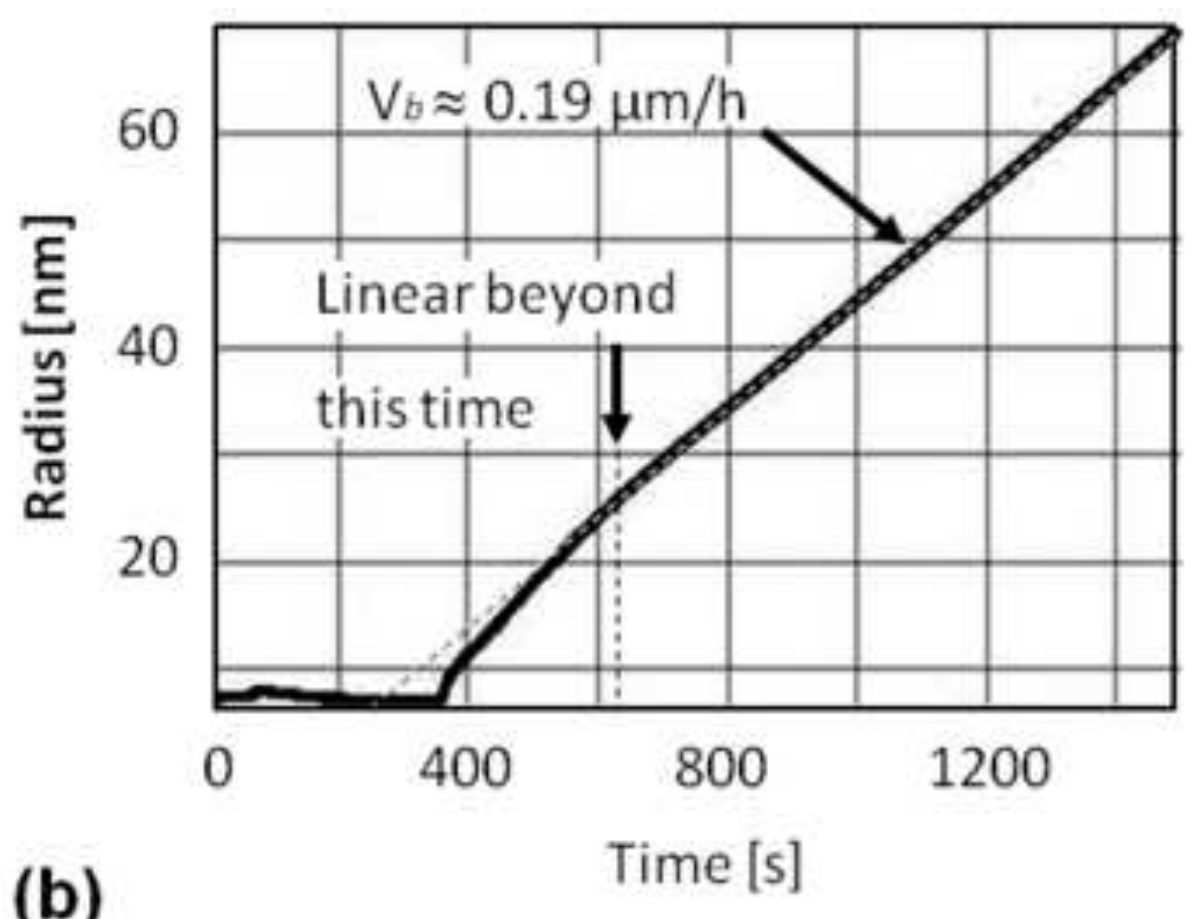



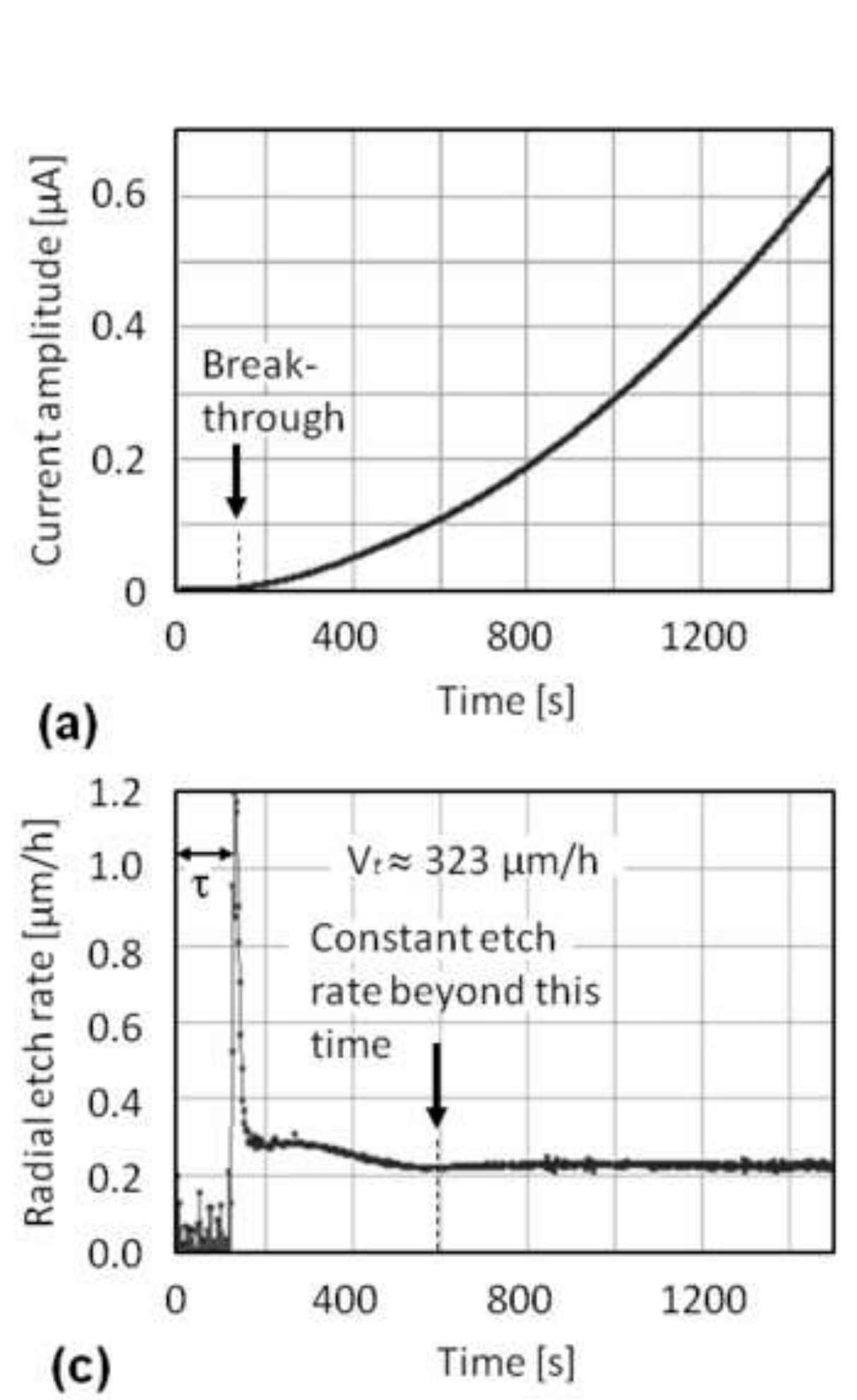

(c) Time [s]
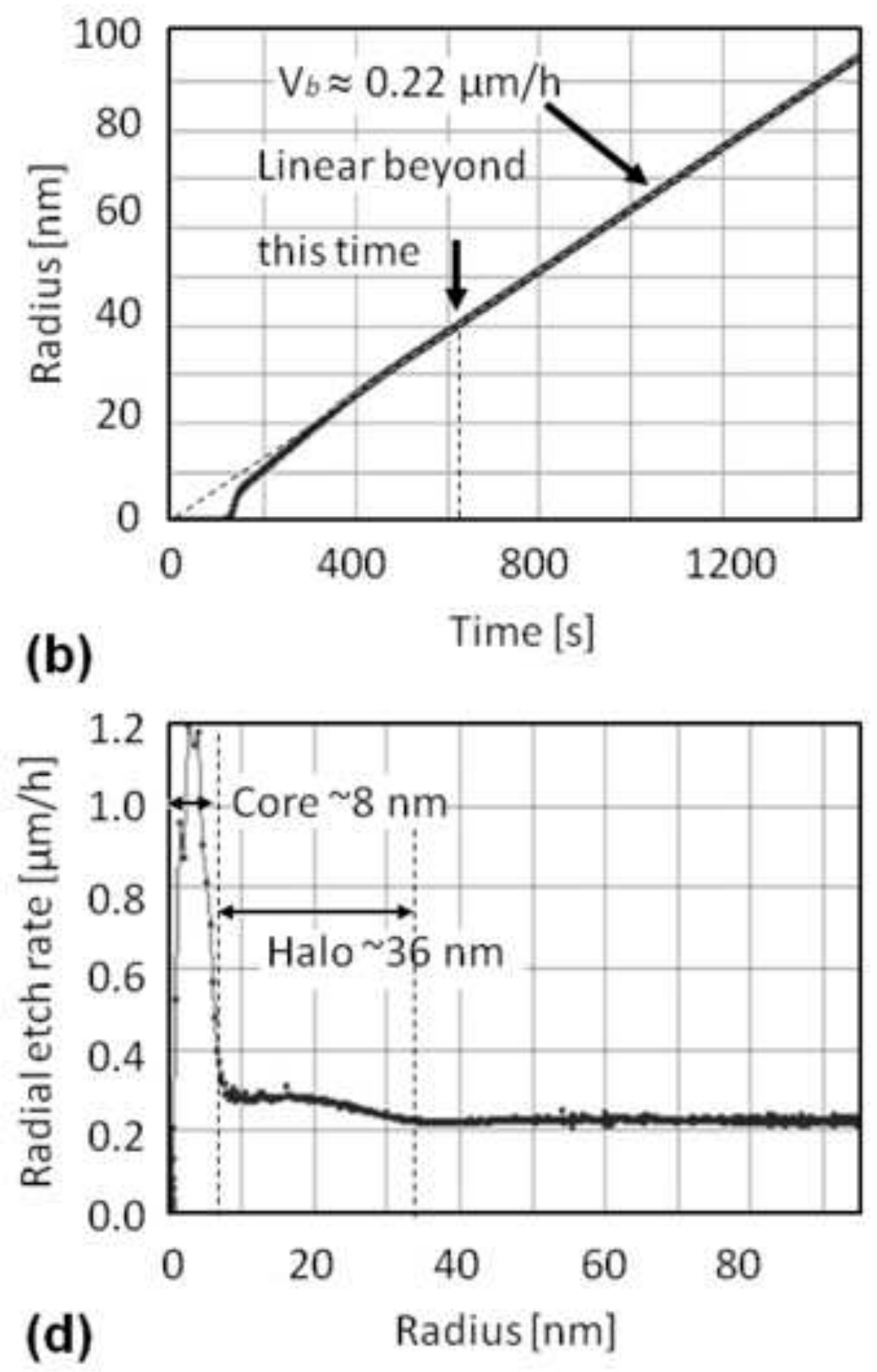
Figure 9-1

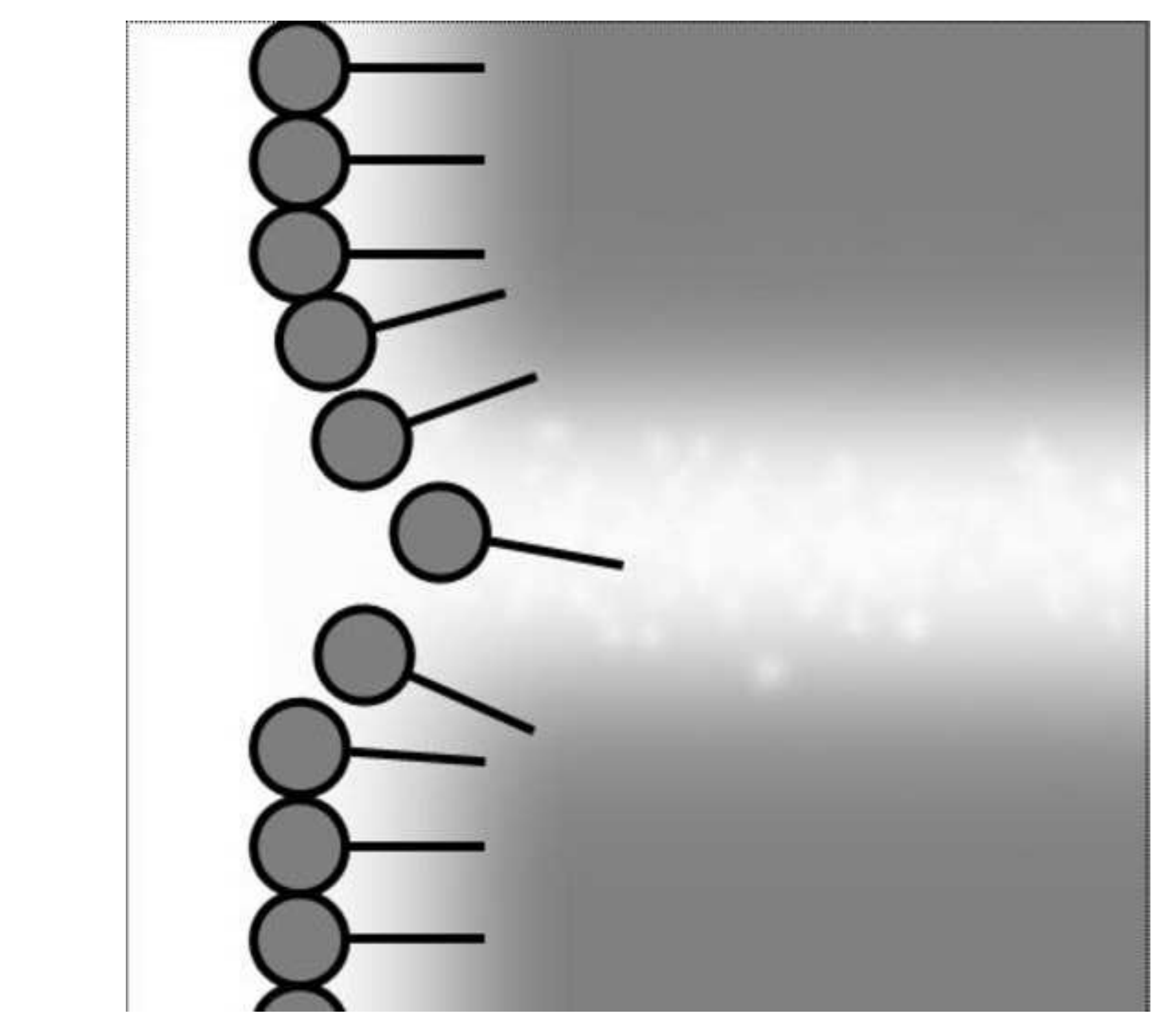

Fure $9-1$

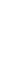


Figure 9-2

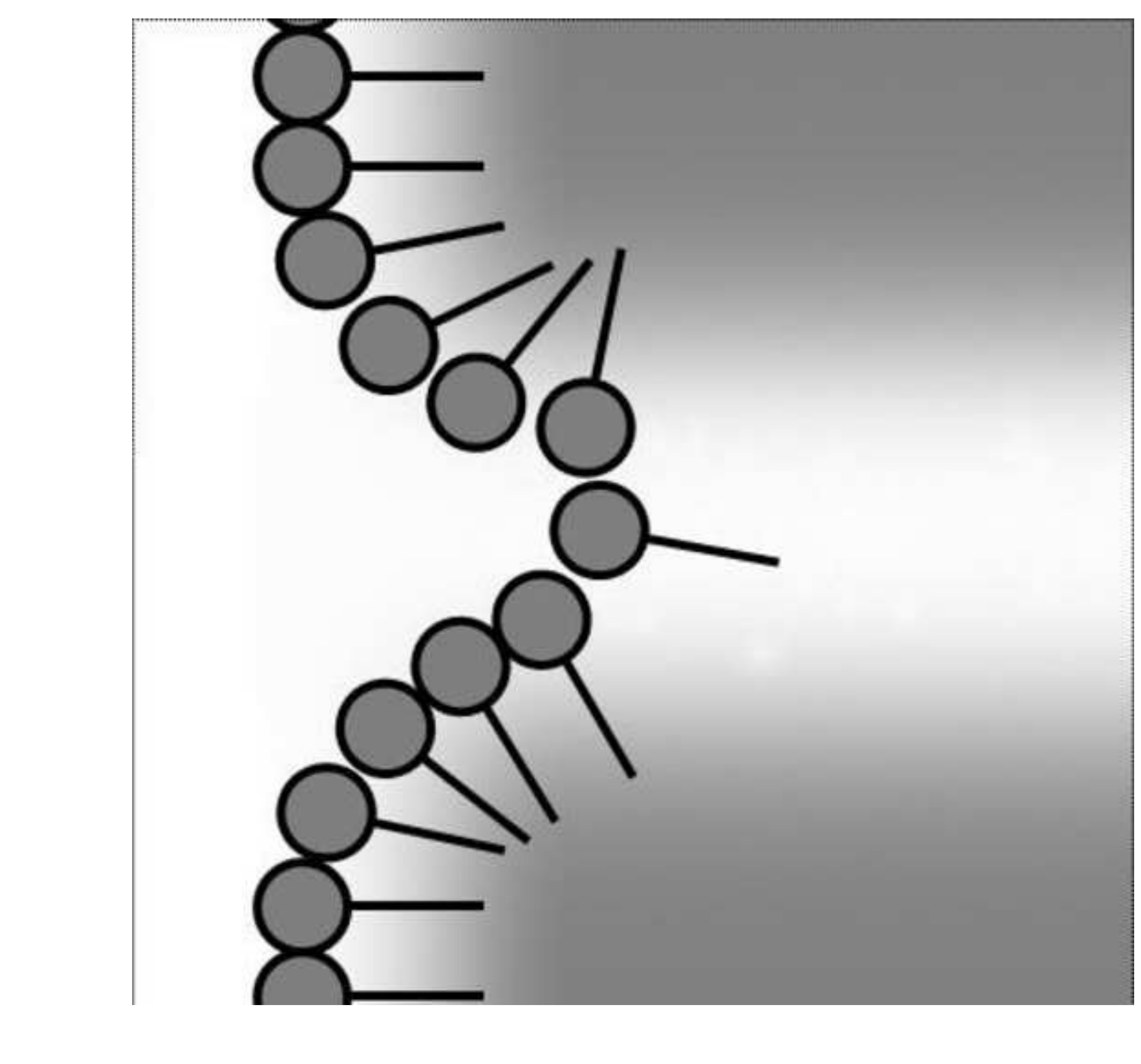

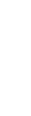

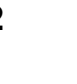
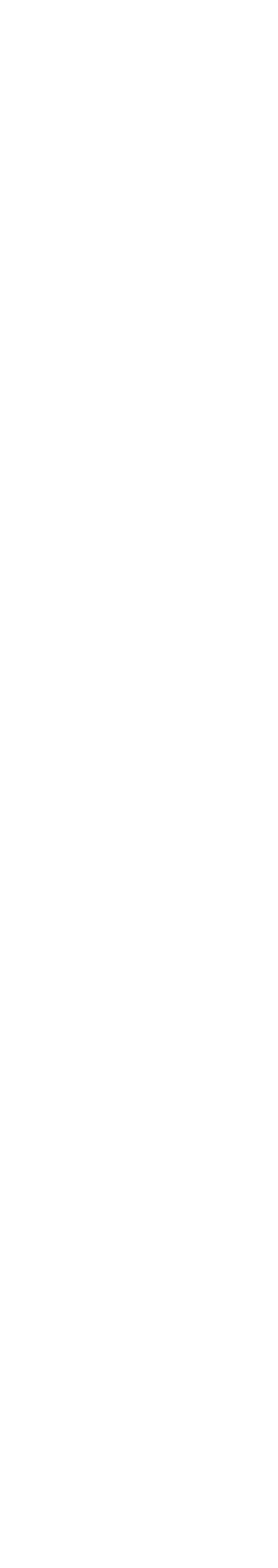


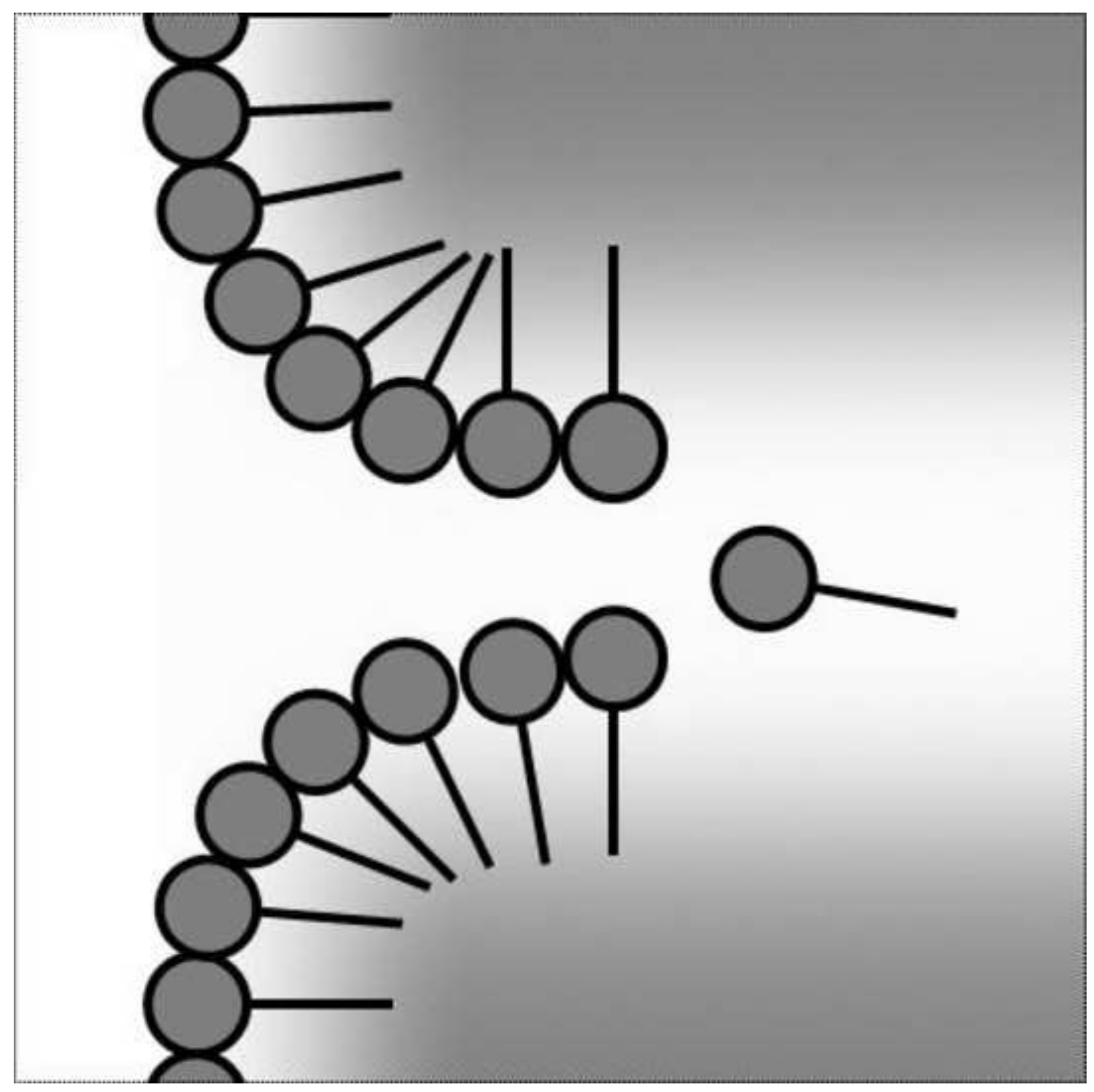




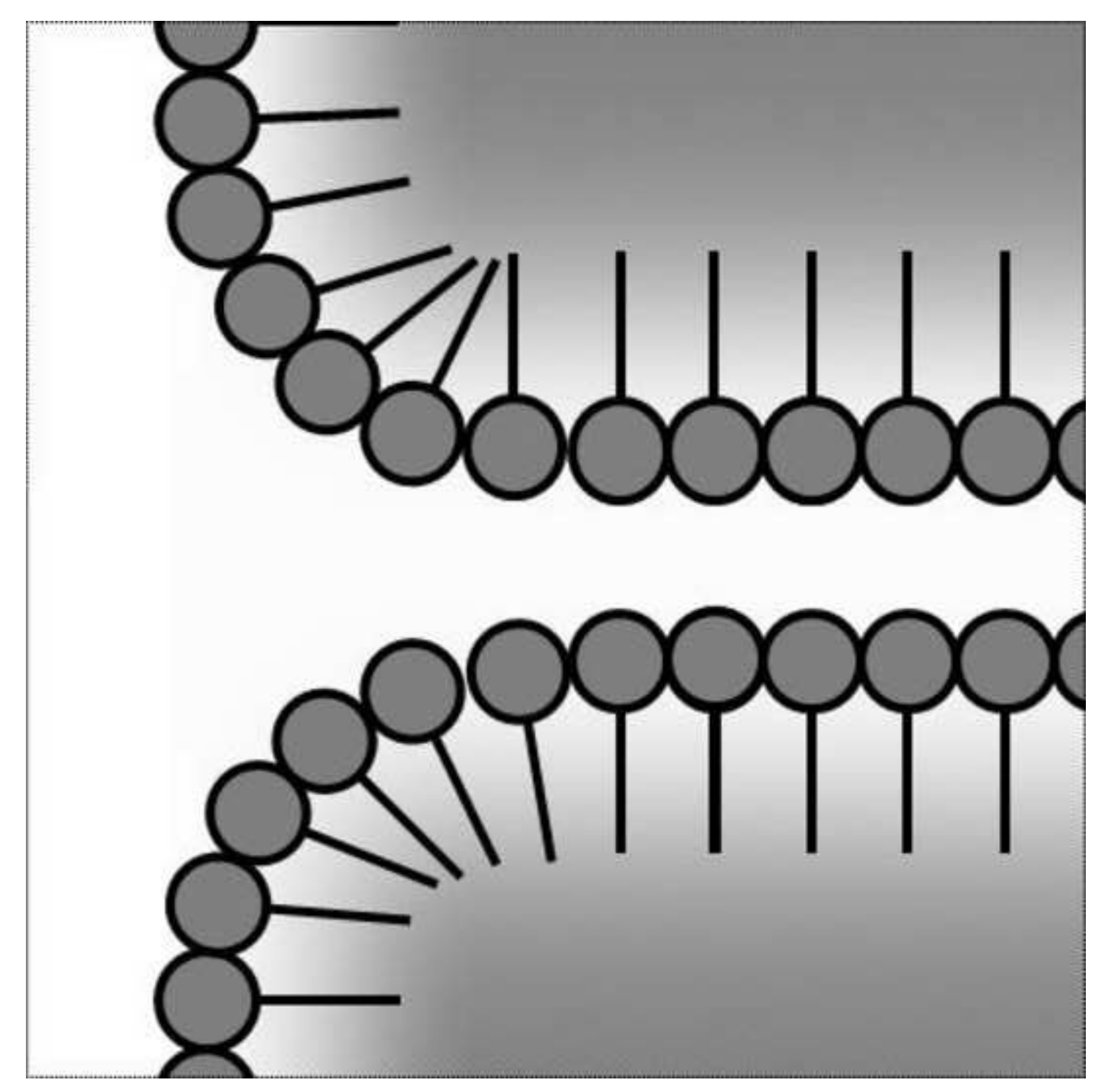




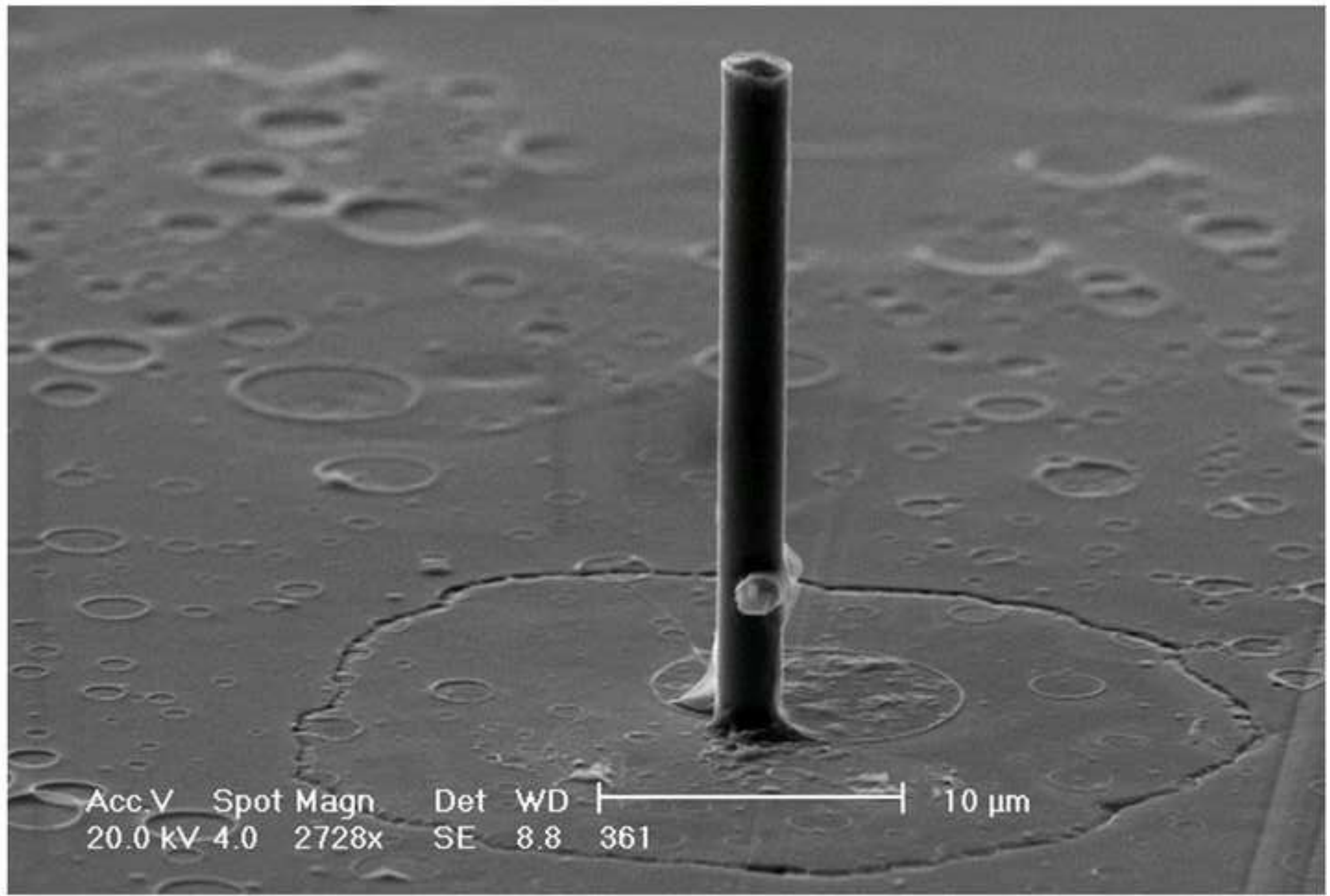




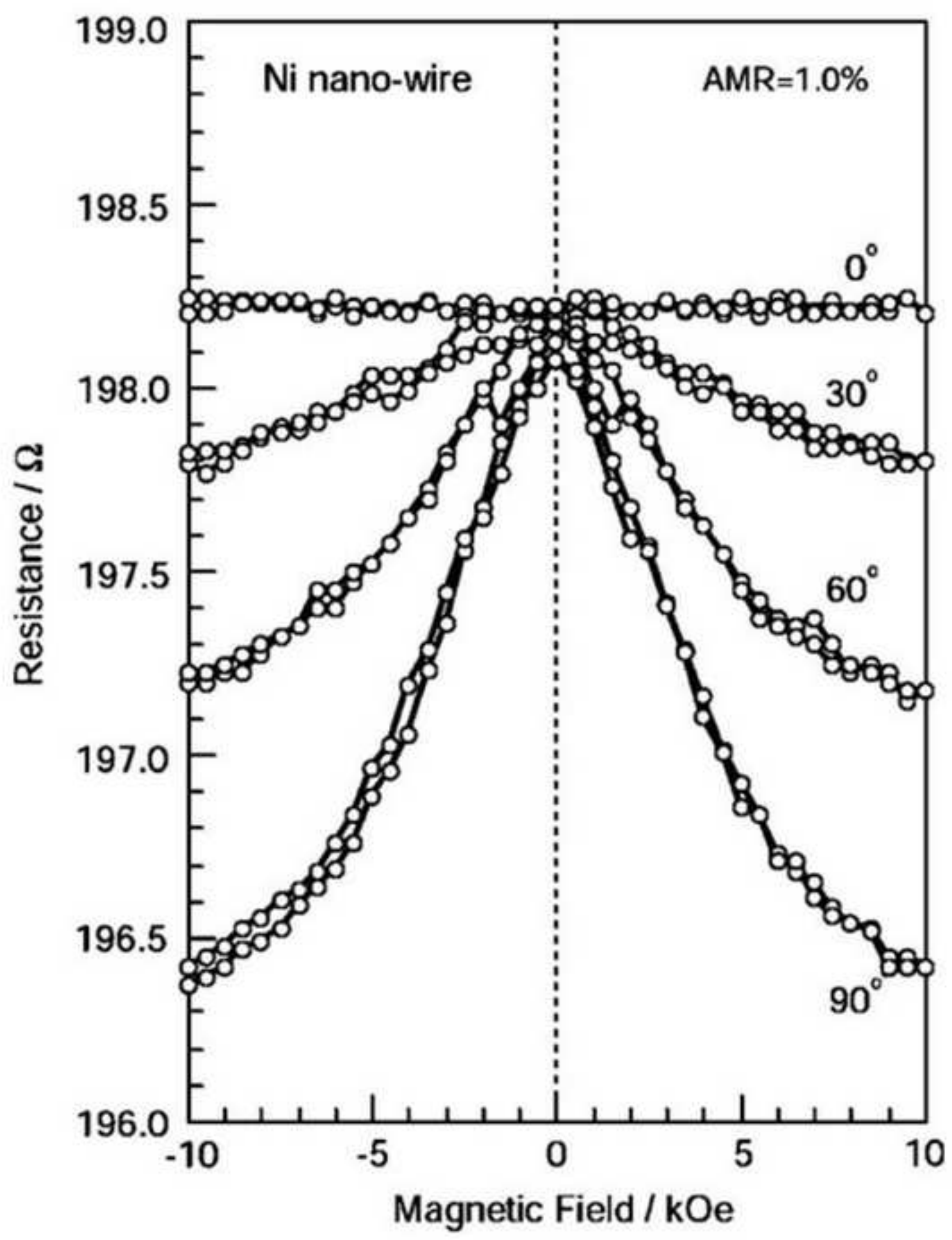

Figure 11 


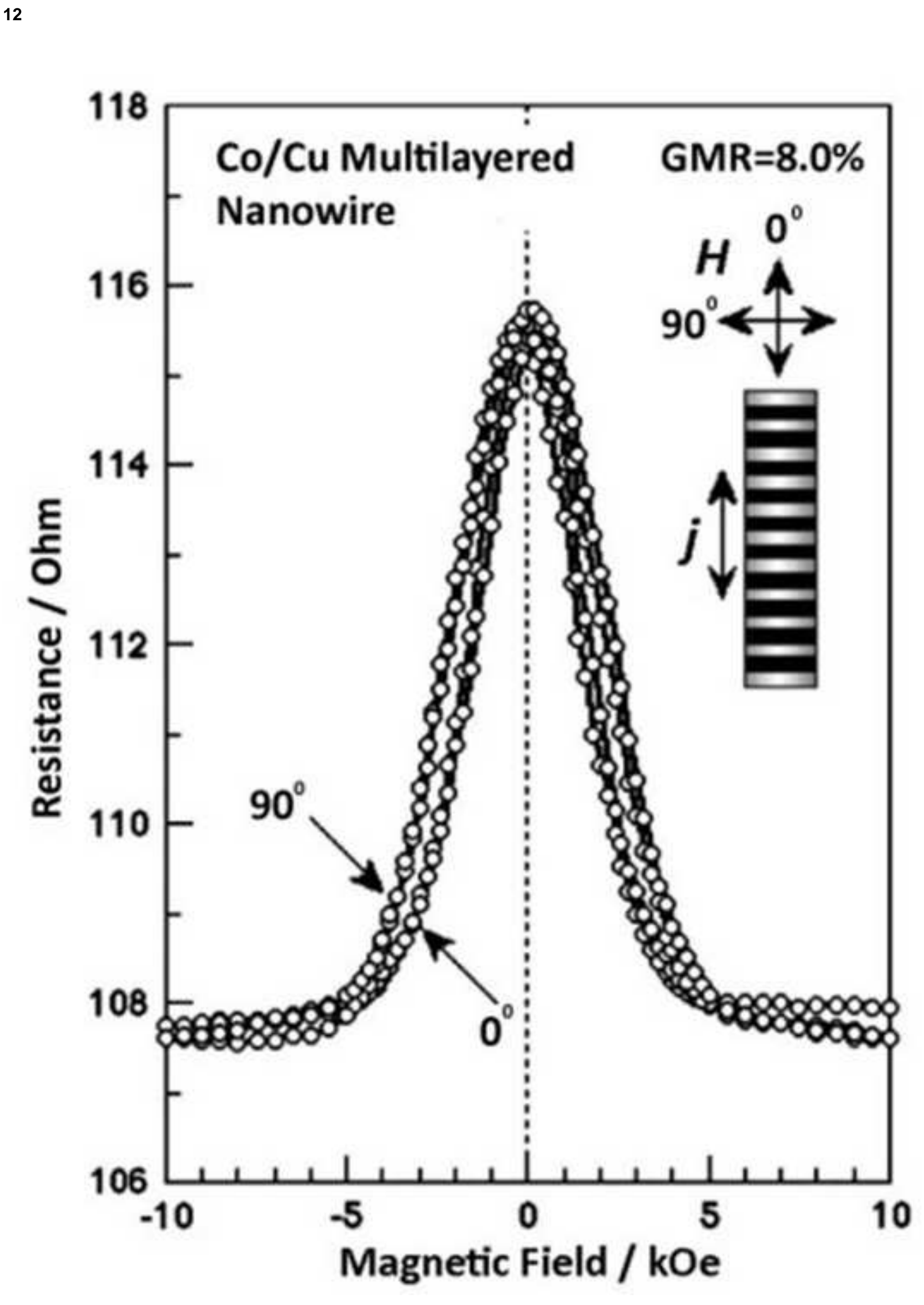

Figure 12

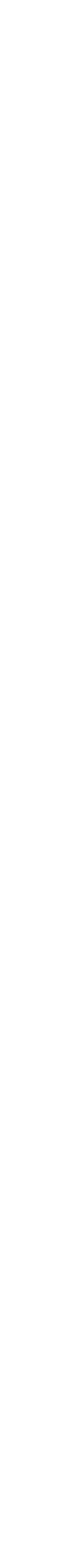
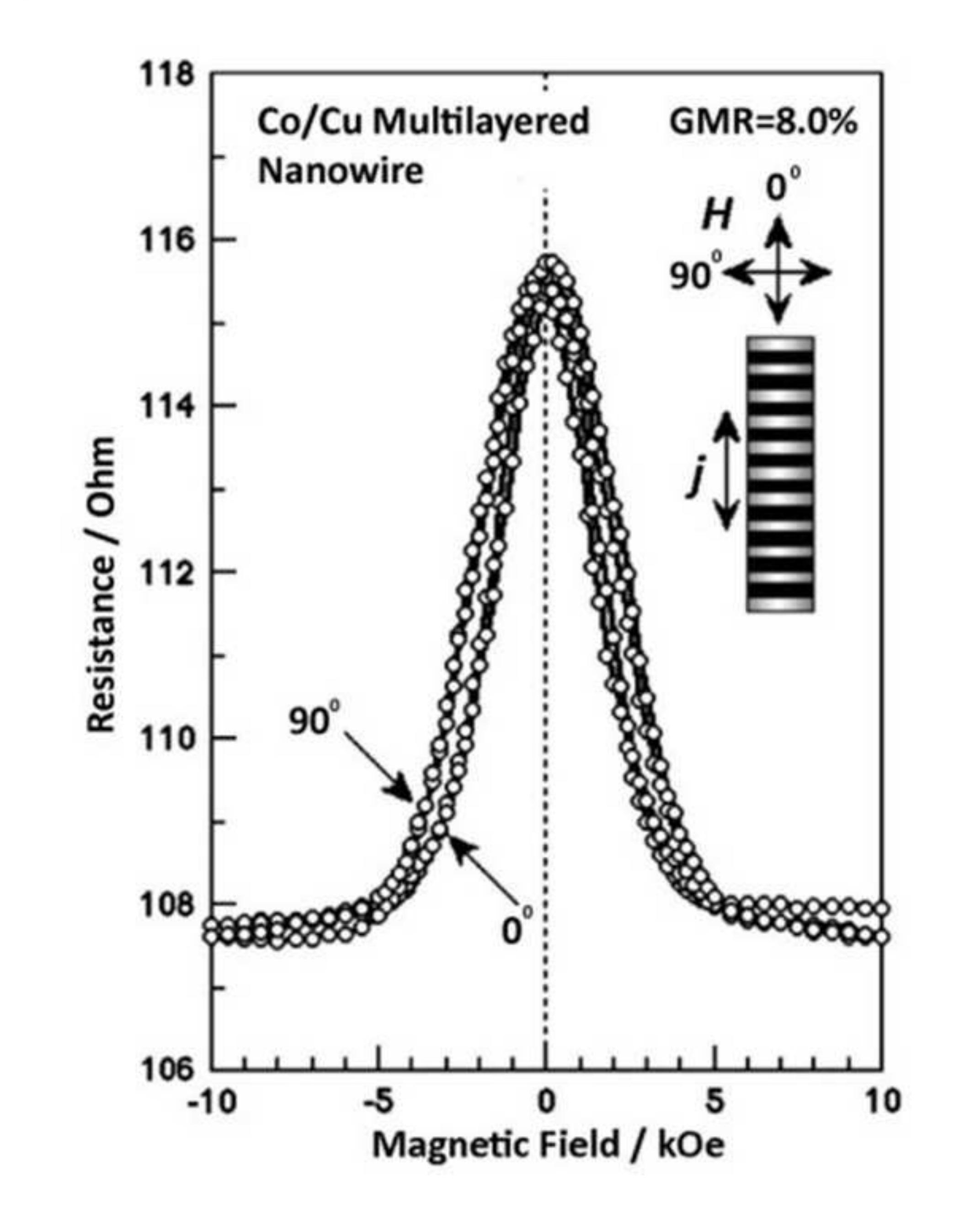

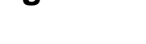 \\ Figure 12}




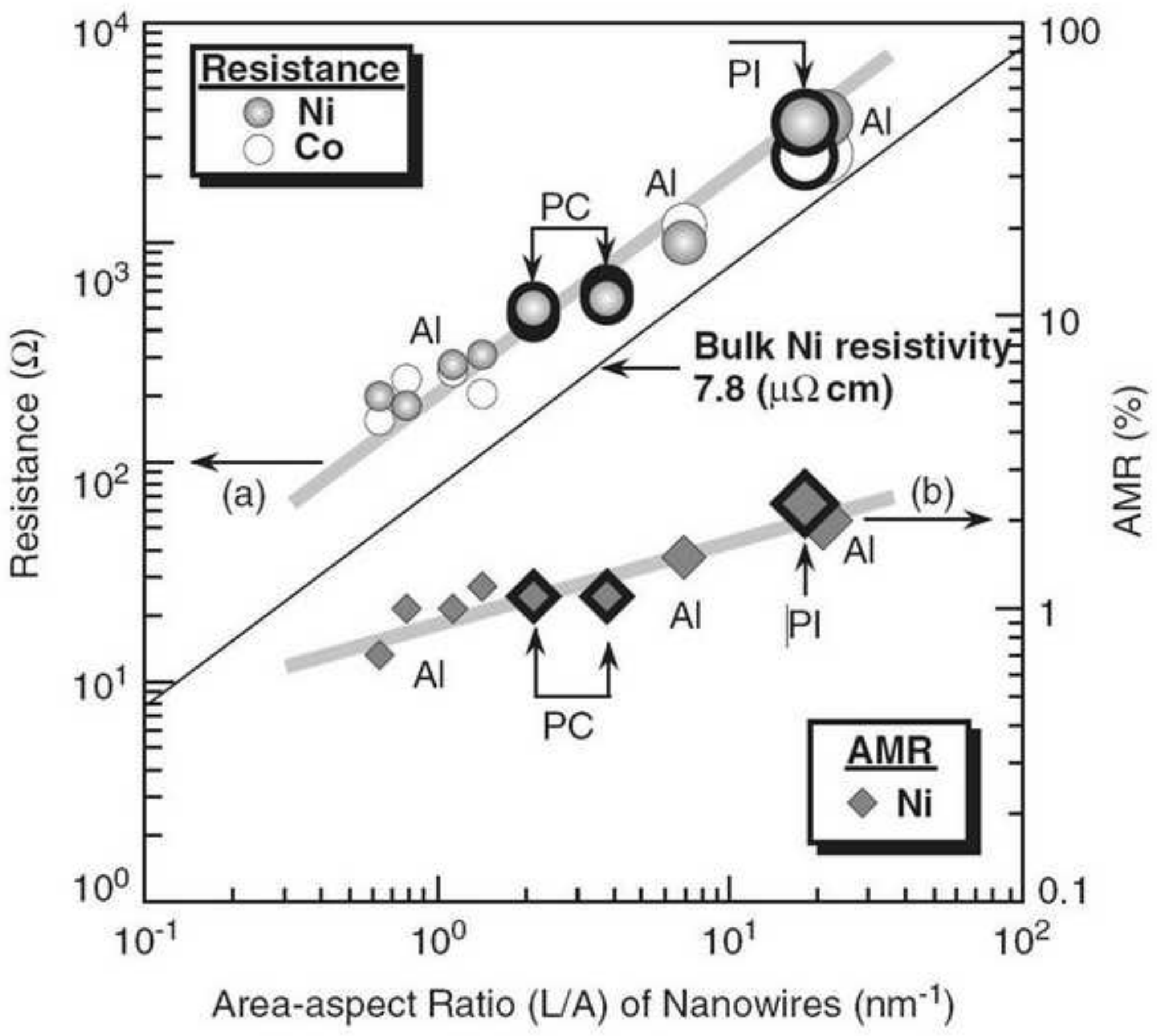

